\title{
Hetero-Diels-Alder Synthesis of 3-Hydroxypyridines: Access to the Nosiheptide Core
}

\author{
Jin-Yong $\mathrm{Lu}^{\dagger}$ and Hans-Dieter Arndt ${ }^{*}, \dagger, \dagger$ \\ ${ }^{\dagger}$ Max-Planck-Institut für molekulare Physiologie, Otto-Hahn-Str. 11, D-44227 \\ Dortmund, Germany, and ${ }^{\ddagger}$ Universität Dortmund, Fachbereich Chemie, \\ Otto-Hahn-Str. 6, D-44221 Dortmund, Germany \\ hans-dieter.arndt@mpi-dortmund.mg.de
}

\section{Supporting Information}

\section{Table of contents}

Table of contents

General methods

Preparation and characterization of 5g, 6c, 6d, 11, 21, 22

Enantiomeric excess determination of building block 23 and 25a 


\section{General methods}

All solvents, when not purchased in suitable purity or dryness, were distilled using standard methods. ${ }^{1}$ Deionized water was used for all experiments. Thin Layer Chromatography(TLC) was carried out on Merck precoated silica gel plates (60F-254) using ultraviolet light irradiation at $254 \mathrm{~nm}$ or the $\mathrm{KMnO}_{4}$ solution $\left(\mathrm{KMnO}_{4} 1 \mathrm{~g}, \mathrm{~K}_{2} \mathrm{CO}_{3}\right.$ $6.6 \mathrm{~g}, 5 \% \mathrm{NaOH}$ solution $1.7 \mathrm{~mL}, \mathrm{H}_{2} \mathrm{O} 100 \mathrm{~mL}$ ) as staining reagent. Silica gel chromatography was performed using silica gel from J. T. Baker or Merck (particle size 40-60 $\mu \mathrm{m}$ ) under approximately 0.5 bar pressure. ${ }^{1} \mathrm{H}$ - and ${ }^{13} \mathrm{C}-\mathrm{NMR}$ spectra were recorded using a Varian Mercury 400 spectrometer $\left(400 \mathrm{MHz}\left({ }^{1} \mathrm{H}\right)\right.$ and $100.6\left({ }^{13} \mathrm{C}\right)$. Chemical shifts are expressed in parts per million ( $\mathrm{ppm})$ and the spectra are calibrated to residual solvent signals of $\mathrm{CDCl}_{3}(7.26 \mathrm{ppm}$ and $77.0 \mathrm{ppm}), \mathrm{CD}_{3} \mathrm{CN}$ (1.94 ppm and $1.24 \mathrm{ppm}$ ), DMSO (2.50 ppm and $39.43 \mathrm{ppm}$ ). Coupling constants are given in Hertz (Hz) and the following notations indicate the multiplicity of the signals: $s$ (singlet), $d$ (doublet), $t$ (triplet), q (quartet), m (multiple), br (broad signal).

Matrix assisted laser desorption ionization time-of-flight (MALDI-TOF) measurements were carried out with Voyager-DE Pro Biospectrometry. Workstation from PerSeptive Biosystems using 2,5-dihydrobenzoic acid as matrix (unless stated otherwise). High resolution mass spectra (HRMS, $70 \mathrm{ev)}$ were measured on a Jeol SX 102 spectrometer by using electron impact (EI), fast atom bombardment (FAB) techniques with $m$-nitrobenzylalcohol as a matrix. Calculated masses were obtained by using the software ChemDraw Ultra (CambridgeSoft Corporation).

Gas chromatography - mass spectrometry (GC-MS) Spectra were obtained from a Hewlett Packard 6890 GC system coupled to a Hewlett Packard 5973 mass selective Detector. A HP 5TA capillary column $(0.33 \mu \mathrm{m}$ x $25 \mathrm{~m}$ x $0.2 \mathrm{~mm})$ and helium flow rate of $2 \mathrm{~mL} / \mathrm{min}$ were used. Method A: temperature gradient: 0 min $\left(100^{\circ} \mathrm{C}\right) \rightarrow 1 \mathrm{~min}$ $\left(100^{\circ} \mathrm{C}\right) \rightarrow 6 \min \left(300^{\circ} \mathrm{C}\right) \rightarrow 12 \min \left(300^{\circ} \mathrm{C}\right)$. Method $B$ : temperature gradient: 0 min $\left(50^{\circ} \mathrm{C}\right) \rightarrow 2 \min \left(50^{\circ} \mathrm{C}\right) \rightarrow 8 \min \left(300^{\circ} \mathrm{C}\right) \rightarrow 14 \min \left(300^{\circ} \mathrm{C}\right)$. 
Fourier transform infrared spectroscopy (FT-IR) spectra were obtained with a Bruker vector 22 spectrometer with a diffuse reflectance head A527 from Spectra Tech $(\mathrm{KBr}$ as matrix). The following notations indicate the intensity of the absorption bands: $\mathrm{s}=$ strong, $\mathrm{m}=$ middle, $\mathrm{w}=$ weak, $\mathrm{b}=$ broad .

Optical rotations were measured in a Schmidt + Haensch Polartronic HH8 polarimeter at $589 \mathrm{~nm}$. Concentrations are given in $\mathrm{g} / 100 \mathrm{~mL}$ solvent.

Melting points were determined in a Büchi melting point B-540 apparatus in open capillaries (uncorrected).

All starting material and reagents were from commercial suppliers (Acros, Aldrich, Novabiochem, Fluka) and used without purification, with the following exceptions, which were prepared according to published procedures: oxime 30,1-aza-1,3-butadienes $\mathbf{6 a}$ and $\mathbf{6 b}$, all halogenated alkynes, and bromothiazole $\mathbf{1 6}^{2,3,4,5}$

\section{1-Phenylprop-2-yn-1-one (5n)}

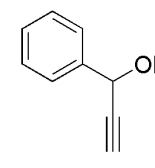

29

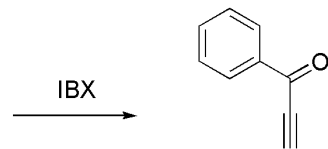

$5 n$

To a cooled solution $\left(0^{\circ} \mathrm{C}\right)$ of 1-phenyl-2-propyn-1-ol $29(0.5 \mathrm{~g}, 3.8 \mathrm{mmol})$ in dry THF $(10 \mathrm{~mL})$ under argon was added dropwise a mixture of IBX (1.27 g, $4.5 \mathrm{mmol})$ in dry DMSO $(10 \mathrm{~mL})$. The solution was allowed warm to room temperature after $2 \mathrm{~h}$ and stirred for $1 \mathrm{~h}$ (TLC control). The mixture was diluted with water $(60 \mathrm{~mL})$ and extracted with diethyl ether $(4 \times 50 \mathrm{~mL})$. The combined extracts were dried over sodium sulfate and evaporated to dryness. Purification by column chromatography on silica gel (EtOAc/cyclohexane $=1: 10)$ gave $0.47 \mathrm{~g}(3.6 \mathrm{mmol}, 96 \%)$ ketone $5 \mathbf{n}$ as a colorless solid. M. p. $=53-55^{\circ} \mathrm{C}$. TLC: $R_{f}=0.53($ EtOAc/cyclohexane $=1: 2)$. GC-MS (method 
B): $T_{R}=5.12 \mathrm{~min}, 130\left(\mathrm{M}^{+}\right) .{ }^{1} \mathrm{H}-\mathrm{NMR}\left(400 \mathrm{MHz}, \mathrm{CD}_{3} \mathrm{CN}\right) \delta=3.92(1 \mathrm{H}, \mathrm{s}, \mathrm{CH})$, 7.54$7.58(2 \mathrm{H}, \mathrm{m}, \mathrm{Ar}), 7.69-7.72(1 \mathrm{H}, \mathrm{m}, \mathrm{Ar}), 8.13-8.15(2 \mathrm{H}, \mathrm{m}, \mathrm{Ar}) .{ }^{13} \mathrm{C}-\mathrm{NMR}(100.6 \mathrm{MHz}$, $\left.\mathrm{CD}_{3} \mathrm{CN}\right) \delta=80.68(\underline{\mathrm{CCH}}), 82.96(\underline{\mathrm{CH}}), 129.85(\mathrm{Ar}), 130.24(\mathrm{Ar}), 135.63(\mathrm{Ar}), 137.10$ (Ar), $178.24(\mathrm{C}(\mathrm{O}) \mathrm{Ar}) . \mathrm{IR}(\mathrm{KBr}): v=3238 \mathrm{~s}, 2095 \mathrm{~s}, 1662 \mathrm{~m}, 697 \mathrm{~s} \mathrm{~cm}^{-1}$.

\section{2-Methoxycarbonyl-1,3-bis(triethylsiloxy)-1-aza-1,3-butadiene (6c)}

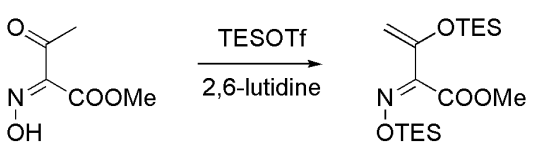

30

$6 \mathrm{c}$

Triethylsilyl triflate $(5.68 \mathrm{~mL}, 25 \mathrm{mmol})$ was added dropwise to a stirred solution of methyl oximino-acetoacetate $30(1.81 \mathrm{~g}, 12.5 \mathrm{mmol})$ and 2,6-lutidine $(5.80 \mathrm{~mL}$, $50 \mathrm{mmol})$ in dry dichloromethane $(15 \mathrm{~mL})$ at $0^{\circ} \mathrm{C}$ under an argon. The mixture was allowed to warm to room temperature after $1 \mathrm{~h}$ and stirred for $12 \mathrm{~h}$. Satured $\mathrm{NaCl}$ solution (40 mL) was added and the mixture was extracted with dichloromethane ( 3 x $60 \mathrm{~mL}$ ). The combined organic extracts were dried with $\mathrm{Na}_{2} \mathrm{SO}_{4}$, concentrated to dryness and purified by column chromatography on silica gel $($ EtOAc/cyclohexane $=1: 12)$ to give $4.39 \mathrm{~g}(11.8 \mathrm{mmol}, 92 \%) 1$-azadiene $6 \mathrm{c}$ as a colorless oil. TLC: $R_{f}=0.84($ EtOAc/cyclohexane $=1: 2)$. GC-MS (method A): $T_{R}=7.45 \mathrm{~min}, 373\left(\mathrm{M}^{+}\right)(95 \%), T_{R}=7.39 \mathrm{~min}, 373\left(\mathrm{M}^{+}\right)(5 \%) .{ }^{1} \mathrm{H}-\mathrm{NMR}(400 \mathrm{MHz}$, $\left.\mathrm{CDCl}_{3}\right) \delta=0.71-0.97(30 \mathrm{H}, \mathrm{m}, \mathrm{TES}), 3.83(3 \mathrm{H}, \mathrm{s}, \mathrm{COOMe}), 4.64(1 \mathrm{H}, \mathrm{d}, J=2.0$, $\left.\mathrm{C}=\mathrm{CH}_{2}\right), 4.67\left(1 \mathrm{H}, \mathrm{d}, J=2.0, \mathrm{C}=\mathrm{CH}_{2}\right) \cdot{ }^{13} \mathrm{C}-\mathrm{NMR}\left(100.6 \mathrm{MHz}, \mathrm{CDCl}_{3}\right) \delta=4.42$ $\left(\mathrm{C}-\mathrm{OSiC} \underline{H}_{2}\right), 4.98(\mathrm{~N}-\mathrm{OSi} \underline{\mathrm{CH}} 2), 6.60\left(\mathrm{C}-\mathrm{OSiCH}_{2} \underline{\mathrm{CH}}_{3}\right), 6.68\left(\mathrm{~N}-\mathrm{OSiCH}_{2} \underline{\mathrm{CH}}_{3}\right), 52.11$ $\left(\mathrm{COOCH}_{3}\right), 99.63\left(\mathrm{C}=\underline{\mathrm{CH}}_{2}\right), 148.92\left(\underline{\mathrm{C}}=\mathrm{CH}_{2}\right), 154.92$ (ㄷ-COOMe), 164.09(COOMe). IR $(\mathrm{KBr}): v=2960 \mathrm{~s}, 2736 \mathrm{~s}, 1757 \mathrm{~s}, 1600 \mathrm{~s}, 942 \mathrm{~s} \mathrm{~cm}^{-1}$. 


\section{2-(4-Ethoxycarbonyl-thiazole-)-1,3-bis(triethylsiloxy)-1-aza-1,3-butadiene (6d)}

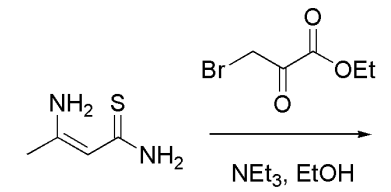

31

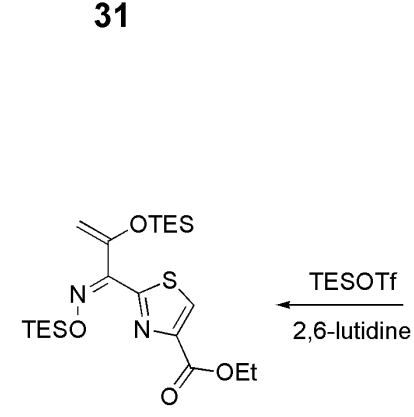

6d

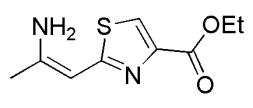

32
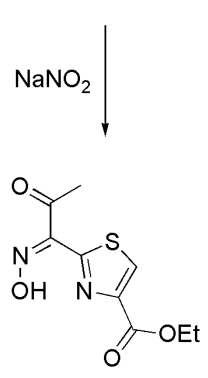

33

A mixture of aminocrotonic thioamide $31(1.16 \mathrm{~g}, 10 \mathrm{mmol})$, ethyl bromopyruvate $(1.4 \mathrm{~mL}, 13 \mathrm{mmol})$, triethylamine $(2.07 \mathrm{~mL}, 15 \mathrm{mmol})$ and ethanol $(5 \mathrm{~mL})$ was heated to reflux for exactly $20 \mathrm{~min}$ (TLC control) and cooled quickly to room temperature. Water $(100 \mathrm{~mL})$ was added, the solid precipitate was filtered off and recrystallized from isopropanol (5 mL) to give $1.6 \mathrm{~g}(7.5 \mathrm{mmol}, 76 \%)$ of thiazole 32 as a yellow solid.

M. p. $=109-110^{\circ} \mathrm{C}$ (isopropanol). TLC: $R_{f}=0.17$ (EtOAc/cyclohexane $\left.=1: 2\right)$. GC-MS

$(\operatorname{method} \mathrm{B}): T_{R}=7.21 \mathrm{~min}, 212\left(\mathrm{M}^{+}\right)$. NMR: ${ }^{1} \mathrm{H}-\mathrm{NMR}\left(400 \mathrm{MHz}, \mathrm{CDCl}_{3}\right) \delta=1.36-1.39$ $\left(3 \mathrm{H}, \mathrm{t}, J=7.0,-\mathrm{CH}_{2} \underline{\mathrm{CH}_{3}}\right), 1.99\left(3 \mathrm{H}, \mathrm{s}, \mathrm{CH}_{3}\right), 4.33-4.49\left(2 \mathrm{H}, \mathrm{q}, J=7.2,-\underline{\mathrm{CH}}_{2} \mathrm{CH}_{3}\right), 5.18$ $(1 \mathrm{H}, \mathrm{s}, \mathrm{CH}=\mathrm{C}), 6.46\left(2 \mathrm{H}, \mathrm{s}, \mathrm{NH}_{2}\right), 7.70(1 \mathrm{H}, \mathrm{s}, \mathrm{CH}) .{ }^{13} \mathrm{C}-\mathrm{NMR}\left(100.6 \mathrm{MHz}, \mathrm{CDCl}_{3}\right)$ $\left.\delta=14.35\left(\mathrm{CH}_{2} \underline{\mathrm{CH}}\right)_{3}\right), 22.19\left(\underline{\mathrm{CH}_{3}}\right), 61.0\left(\underline{\mathrm{CH}}_{2} \mathrm{CH}_{3}\right), 88.33(\underline{\mathrm{CH}}=\mathrm{C}), 121.16(\underline{\mathrm{CH}}), 145.97$ (ㄷ-COOEt), $149.57\left(\underline{\mathrm{C}}-\mathrm{NH}_{2}\right), 161.61(\underline{\mathrm{C}}(\mathrm{N}) \mathrm{S}), 168.80\left(\underline{\left.\mathrm{COOCH}_{2} \mathrm{CH}_{3}\right) .} \mathrm{IR}(\mathrm{KBr})\right.$ : $v=3410 \mathrm{~s}, 3100 \mathrm{~s}, 2980 \mathrm{~s}, 2903 \mathrm{~m}, 1715 \mathrm{~s}, 1633 \mathrm{w}, 1219 \mathrm{~s}, 800 \mathrm{~s} \mathrm{~cm}^{-1}$. HRMS (FAB), $\left[\mathrm{M}^{+}\right]$, Calc: 212.0619, found: 212.0607. Anal. Calcd for $\mathrm{C}_{9} \mathrm{H}_{12} \mathrm{~N}_{2} \mathrm{O}_{2} \mathrm{~S}: \mathrm{C}, 50.92 ; \mathrm{H}, 5.70$; N, 13.20; found: C, 50.4; H, 5.6; N, 12.6.

Sodium nitrite $(0.16 \mathrm{~g}, 2.3 \mathrm{mmol})$ in water $(5 \mathrm{~mL})$ was added dropwise to a cooled thiazole $32(0.48 \mathrm{~g}, 2.3 \mathrm{mmol})$ in glacial acetic acid $(5 \mathrm{~mL})$ and water $(5 \mathrm{~mL})$. The mixture was stirred for $30 \mathrm{~min}$ and $2 \mathrm{~h}$ at room temperature. The mixture was diluted 
with water $(100 \mathrm{~mL})$ and extracted with diethyl ether $(3 \times 50 \mathrm{~mL})$. The ethereal layers were combined, washed with water $(100 \mathrm{~mL})$, saturated sodium bicarbonate $(4 \times 50 \mathrm{~mL})$ and brine $(50 \mathrm{~mL})$. The ether layer was dried with sodium sulfate and concentrated to dryness. Purification by column chromatography on silica gel (EtOAc/cyclohexane $=1: 6)$ delivered $0.34 \mathrm{~g}(1.4 \mathrm{mmol}, 60 \%)$ of oxime 33 as a yellow solid. M. p. $=120-121^{\circ} \mathrm{C}$. TLC: $R_{f}=0.33$ (EtOAc/cyclohexane $=1: 2$ ). GC-MS (method B): $T_{R}=7.28 \mathrm{~min}, 242\left(\mathrm{M}^{+}\right)$. NMR: ${ }^{1} \mathrm{H}-\mathrm{NMR}\left(400 \mathrm{MHz}, \mathrm{CDCl}_{3}\right) \delta=1.39-1.42(3 \mathrm{H}, \mathrm{t}$, $\left.J=7.2, \mathrm{CH}_{2} \underline{\mathrm{CH}}_{3}\right), 2.61\left(3 \mathrm{H}, \mathrm{s}, \mathrm{CH}_{3}\right), 4.39-4.45\left(2 \mathrm{H}, \mathrm{q}, J=7.0, \underline{\mathrm{CH}}_{2} \mathrm{CH}_{3}\right), 8.36(1 \mathrm{H}, \mathrm{s}$, $\mathrm{CH}) .{ }^{13} \mathrm{C}-\mathrm{NMR}\left(100.6 \mathrm{MHz}, \mathrm{CDCl}_{3}\right) \delta=14.22\left(\mathrm{CH}_{2} \mathrm{CH}_{3}\right), 24.74\left(\underline{\mathrm{CH}}_{3}\right), 61.89$ $\left(\underline{\mathrm{C}} \mathrm{H}_{2} \mathrm{CH}_{3}\right), 130.61(\underline{\mathrm{C}} \mathrm{H}), 143.67$ (ㅁ-COOEt), $144.45(\underline{\mathrm{C}}(\mathrm{N}) \mathrm{OH}), 155.07(\underline{\mathrm{C}}(\mathrm{N}) \mathrm{S}), 159.85$ $\left(\underline{\mathrm{COOCH}}{ }_{2} \mathrm{CH}_{3}\right), 196.52\left(\underline{\mathrm{C}}(\mathrm{O}) \mathrm{CH}_{3}\right) . \mathrm{IR}(\mathrm{KBr}): v=3100 \mathrm{~s}, 2983 \mathrm{~s}, 2956 \mathrm{~m}, 2529 \mathrm{~b}, 1727$

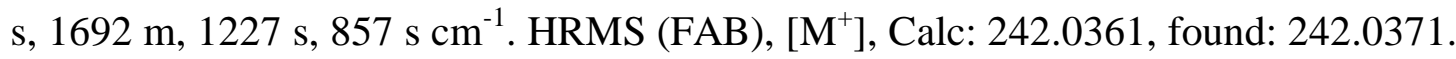

Oxime $33(0.12 \mathrm{~g}, 0.5 \mathrm{mmol})$ gave $0.233 \mathrm{~g}(0.49 \mathrm{mmol}, 99 \%)$ of 1-azadiene $\mathbf{6 d}$ as a yellow oil following the procedure given for $\mathbf{6 c}$.

TLC: $R_{f}=0.65($ EtOAc/cyclohexane $=1: 2)$. GC-MS $\left(\right.$ method B) $: T_{R}=9.14 \mathrm{~min}, 470$ $\left(\mathrm{M}^{+}\right)$. NMR: ${ }^{1} \mathrm{H}-\mathrm{NMR}\left(400 \mathrm{MHz}, \mathrm{CDCl}_{3}\right) \delta=0.51-1.0(30 \mathrm{H}, \mathrm{m}, \mathrm{TES}), 1.35-1.39(3 \mathrm{H}, \mathrm{t}$, $\left.J=7.2, \mathrm{CH}_{2} \underline{\mathrm{CH}}_{3}\right), 4.36-4.41\left(2 \mathrm{H}, \mathrm{q}, J=7.0, \underline{\mathrm{CH}}_{2} \mathrm{CH}_{3}\right), 4.67\left(1 \mathrm{H}, \mathrm{s}, \mathrm{C}=\mathrm{CH}_{2}\right), 4.92(1 \mathrm{H}, \mathrm{s}$, $\left.\mathrm{C}=\mathrm{CH}_{2}\right), 8.31(1 \mathrm{H}, \mathrm{s}, \mathrm{CH}) .{ }^{13} \mathrm{C}-\mathrm{NMR}\left(100.6 \mathrm{MHz}, \mathrm{CDCl}_{3}\right) \delta=4.24\left(\mathrm{C}-\mathrm{OSi}_{\underline{C}} \mathrm{H}_{2}\right), 4.69$ $\left(\mathrm{N}-\mathrm{OSi} \underline{\mathrm{C}} \mathrm{H}_{2}\right), 6.43\left(\mathrm{C}-\mathrm{OSSiCH}_{2} \underline{\mathrm{CH}_{3}}\right), 6.50\left(\mathrm{~N}-\mathrm{OSiCH}_{2} \underline{\mathrm{C}} \mathrm{H}_{3}\right), 14.17\left(\mathrm{CH}_{2} \underline{\mathrm{CH}_{3}}\right), 61.10$ $\left(\underline{\mathrm{CH}}_{2} \mathrm{CH}_{3}\right), 98.10\left(\mathrm{C}=\underline{\mathrm{CH}}_{2}\right), 129.66(\underline{\mathrm{C}} \mathrm{H}), 146.18\left(\underline{\mathrm{C}}=\mathrm{CH}_{2}\right), 151.13(\underline{\mathrm{C}}-\mathrm{COOEt}), 152.58$

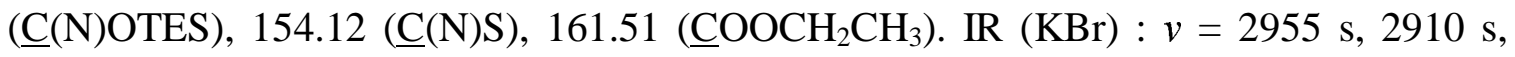
$2877 \mathrm{~s}, 1746 \mathrm{~m}, 1730 \mathrm{~s}, 1715 \mathrm{~m}, 1238 \mathrm{~s}, 857 \mathrm{~s} \mathrm{~cm}^{-1}$.

\section{Azadiene homodimer 11.}

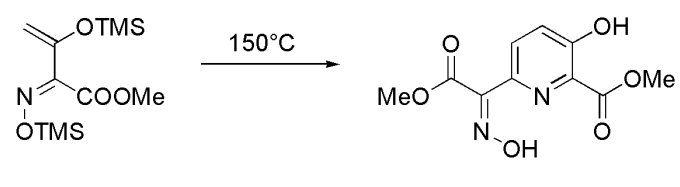

$6 a$ 
1-azadiene 6a $(0.6 \mathrm{~mL}, 2.08 \mathrm{mmol})$ was heated to $150^{\circ} \mathrm{C}$ for $13 \mathrm{~h}$ in a sealed tube. The resulting mixture was purified by column chromatography on silica gel (EtOAc/cyclohexane $=1: 6)$ to give $41 \mathrm{mg}(0.16 \mathrm{mmol}, 15.5 \%)$ of 3-hydroxypyridine $\mathbf{1 1}$ as a colorless solid. M. p. $=157-158^{\circ} \mathrm{C}$. TLC: $R_{f}=0.29($ EtOAc/cyclohexane $=1: 2)$. GC-MS (method B): $T_{R}=7.74 \mathrm{~min}, 254\left(\mathrm{M}^{+}\right) .{ }^{1} \mathrm{H}-\mathrm{NMR}\left(400 \mathrm{MHz}, \mathrm{CDCl}_{3}\right) \delta=3.99$ (3H, s, COOMe), $4.0(3 \mathrm{H}, \mathrm{s}, \mathrm{COOMe}), 7.38,7.40(1 \mathrm{H}, \mathrm{d}, J=8.8, \mathrm{CH}), 7.90,7.93(1 \mathrm{H}, \mathrm{d}$, $J=9.0, \mathrm{CH}), 8.62(1 \mathrm{H}, \mathrm{br}, \mathrm{N}-\mathrm{OH}), 10.90(1 \mathrm{H}, \mathrm{s}, \mathrm{OH}) .{ }^{13} \mathrm{C}-\mathrm{NMR}\left(100.6 \mathrm{MHz}, \mathrm{CDCl}_{3}\right)$ $\delta=52.60\left(\mathrm{COO}^{-} H_{3}\right), 53.11\left(\mathrm{COO}^{-} H_{3}\right), 127.0(\underline{\mathrm{CH}}), 127.06(\underline{\mathrm{CH}}), 129.36(\underline{\mathrm{C}}-\mathrm{COOMe})$, $141.82 \quad(\underline{\mathrm{C}}-\mathrm{C}(\mathrm{N}) \mathrm{OH}), \quad 151.13 \quad(\underline{\mathrm{C}}-\mathrm{OH}), \quad 159.42 \quad(\underline{\mathrm{C}}(\mathrm{N}) \mathrm{OH}), \quad 163.40 \quad(\underline{\mathrm{COOMe}})$, 169.57(COOMe). IR (KBr) : v= $3480 \mathrm{~s}, 3457 \mathrm{~s}, 3159 \mathrm{~b}, 2963 \mathrm{~s}, 2922 \mathrm{~s}, 1745 \mathrm{~s}, 1673 \mathrm{~s}$, 1469 s, 1234 s, 849 s, $804 \mathrm{~s} \mathrm{~cm}^{-1}$. HRMS (ESI), [M+ H $\mathrm{H}^{+}$, calc: 255.0612, found: 255.0612 .

\section{(R)-2-Allyloxycarbonylamino-3-tritylthio-propionic acid (21).}

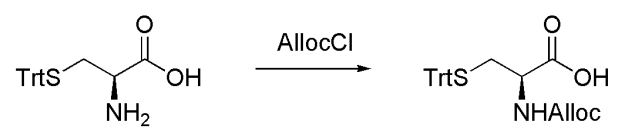

34

A vigorously stirred solution of trityl L-cysteine $34(1.0 \mathrm{~g}, 2.8 \mathrm{mmol})$ in $2 \mathrm{M} \mathrm{NaOH}(1.4$ $\mathrm{mL}, 2.8 \mathrm{mmol})$ was cooled to $0^{\circ} \mathrm{C}$. Allyl chloroformate $(0.35 \mathrm{~mL}, 3.3 \mathrm{mmol})$ and $2 \mathrm{M}$ $\mathrm{NaOH}(1.65 \mathrm{~mL}, 3.3 \mathrm{mmol})$ were added in portions over a period of $10 \mathrm{~min}$. After being stirred at $0^{\circ} \mathrm{C}$ for $1 \mathrm{~h}$, the reaction mixture was acidified to $\mathrm{pH}=2$ with $2 \mathrm{M} \mathrm{HCl}$, and extracted with ethyl acetate $(3 \times 20 \mathrm{~mL})$. The combined organic layers were dried with $\mathrm{Na}_{2} \mathrm{SO}_{4}$ and concentrated. Purification by column chromatography on silica gel $\left(\mathrm{CH}_{2} \mathrm{Cl}_{2} / \mathrm{MeOH}=20: 1\right)$ gave $1.24 \mathrm{~g}(2.77 \mathrm{mmol}, 99 \%)$ of acid 21 as a colorless solid. M. p. $=119-120^{\circ} \mathrm{C}$. TLC: $R_{f}=0.25\left(\mathrm{CH}_{2} \mathrm{Cl}_{2} / \mathrm{MeOH}=10: 1\right) .{ }^{1} \mathrm{H}-\mathrm{NMR}(400 \mathrm{MHz}$, DMSO $\delta=2.38-2.58\left(2 \mathrm{H}, \mathrm{m}, \mathrm{CH}_{2}\right), 3.81-3.86\left(1 \mathrm{H}, \mathrm{d}, J=9.0, \mathrm{CH}_{2} \underline{\mathrm{CH}}\right), 4.46,4.47(2 \mathrm{H}$, d, $\left.J=4.7, \mathrm{COOCH}_{2}\right), 5.16,5.19\left(1 \mathrm{H}, \mathrm{d}, J=10.4, \mathrm{CH}=\mathrm{CH}_{2}\right), 5.28,5.32(1 \mathrm{H}, \mathrm{d}, J=17.3$, $\left.\mathrm{CH}=\underline{\mathrm{CH}_{2}}\right), 5.85-5.93\left(1 \mathrm{H}, \mathrm{m}, \underline{\mathrm{CH}}=\mathrm{CH}_{2}\right), 7.23-7.35(15 \mathrm{H}, \mathrm{m}, \operatorname{trityl}), 7.49,7.51(1 \mathrm{H}, \mathrm{d}$, $J=7.6$, NHCOO $) .{ }^{13} \mathrm{C}-\mathrm{NMR}(100.6 \mathrm{MHz}, \mathrm{DMSO}) \delta=33.09\left(\mathrm{CH}_{2} \mathrm{CH}\right), 53.37$ 
$\left.\left(\mathrm{COO}^{-} H_{2}\right), 64.37(\underline{\mathrm{CPh}})_{3}\right), 66.0\left(\mathrm{CH}_{2} \underline{\mathrm{C}} \mathrm{H}\right), 116.88\left(\mathrm{CH}=\underline{\mathrm{CH}}_{2}\right), 126.66(\mathrm{Ar}), 127.93(\mathrm{Ar})$, 128.98 (Ar), 133.38 (Ar), $144.19\left(\underline{\mathrm{C} H}=\mathrm{CH}_{2}\right), 155.45\left(\underline{\mathrm{COOCH}_{2}}\right), 171.80(\underline{\mathrm{COOH}}) . \mathrm{R}$ $(\mathrm{KBr}): v=3417 \mathrm{~b}, 3061 \mathrm{~s}, 2917 \mathrm{w}, 1730 \mathrm{~s}, 1714 \mathrm{~s}, 1504 \mathrm{~s}, 1445 \mathrm{~s}, 798 \mathrm{~s}, 701 \mathrm{~s} \mathrm{~cm}^{-1}$. $[\alpha]^{20}{ }_{D}=+30.4\left(\mathrm{c}=1.0, \mathrm{CHCl}_{3}\right)$. HRMS (ESI), $\left[\mathrm{M}+\mathrm{Na}^{+}\right]$, Calc: 470.1397, found: 470.1397. Anal. calcd for $\mathrm{C}_{26} \mathrm{H}_{25} \mathrm{NO}_{4} \mathrm{~S}$ : C, 69.78; H, 5.63; N, 3.13; found C, 69.7; H, 6.0; $\mathrm{N}, 3.0$.

\section{(R)-2-Allyloxycarbonylamino-3-tritylthio-propionic thioamide (22).}

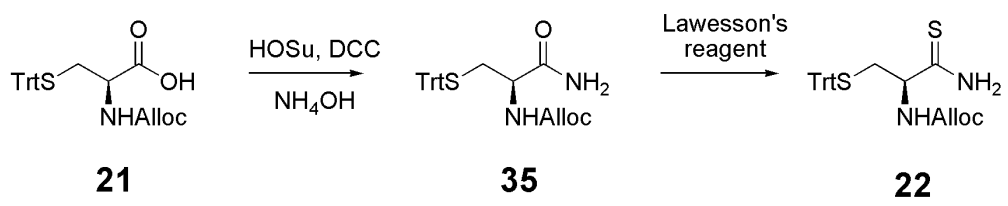

To a stirred solution of acid $21(1.0 \mathrm{~g}, 2.2 \mathrm{mmol})$ in THF $(10 \mathrm{~mL})$ was added HOSu $(0.28 \mathrm{~g}, 2.4 \mathrm{mmol})$ and DCC $(0.51 \mathrm{~g}, 2.4 \mathrm{mmol})$ at $0^{\circ} \mathrm{C}$. The reaction mixture was stirred for $1 \mathrm{~h}$ at $0^{\circ} \mathrm{C}$ and then $5 \mathrm{~h}$ at room temperature. The mixture was filtered and concentrated to dryness. The residue was taken up in ethyl acetate $(20 \mathrm{~mL})$ and cooled to $0^{\circ} \mathrm{C}$, then aqueous $25 \% \mathrm{NH}_{4} \mathrm{OH}$ solution $(0.6 \mathrm{~mL})$ was added dropwise and stirred for $1 \mathrm{~h}$. The mixture was diluted with ethyl acetate $(20 \mathrm{~mL})$, washed with saturated aqueous $\mathrm{NaHCO}_{3}$ solution $(2 \times 10 \mathrm{~mL})$ and brine $(20 \mathrm{~mL})$, dried over $\mathrm{Na}_{2} \mathrm{SO}_{4}$ and concentrated. The resulting residue was purified on silica gel (EtOAc/light petroleum $=1: 2)$ to give $0.99 \mathrm{~g}(2.2 \mathrm{mmol}, 99 \%)$ of the primary amide 35 as a colorless solid. M. p. $=96-98^{\circ} \mathrm{C}$. TLC: $R_{f}=0.21($ EtOAc/cylohexane $=1: 2) .{ }^{1} \mathrm{H}-\mathrm{NMR}(400 \mathrm{MHz}, \mathrm{DMSO}) \delta=4.10-4.15$ $\left(2 \mathrm{H}, \mathrm{m}, J=6.8, \mathrm{CH}_{2}\right), 4.57-4.58\left(2 \mathrm{H}, \mathrm{t}, J=3.7, \mathrm{COOCH}_{2}\right), 5.25,5.28(1 \mathrm{H}, \mathrm{d}, J=10.6$, $\left.\mathrm{CH}=\underline{\mathrm{CH}_{2}}\right), 5.38,5.42\left(1 \mathrm{H}, \mathrm{d}, J=17.2, \mathrm{CH}=\underline{\mathrm{CH}}_{2}\right), 5.97-6.04\left(1 \mathrm{H}, \mathrm{m}, J=5.3, \underline{\mathrm{CH}}=\mathrm{CH}_{2}\right)$, $7.22\left(1 \mathrm{H}, \mathrm{s}, \mathrm{CH}_{2} \mathrm{CH}\right), 7.33,7.34\left(2 \mathrm{H}, \mathrm{d}, J=4.1, \mathrm{NH}_{2}\right), 7.41-7.42(15 \mathrm{H}, \mathrm{m}$, trityl), 7.47, $7.49(1 \mathrm{H}, \mathrm{d}, J=8.4, \mathrm{NH}) .{ }^{13} \mathrm{C}-\mathrm{NMR}(100.6 \mathrm{MHz}, \mathrm{DMSO}) \delta=33.96\left(\underline{\mathrm{CH}}_{2} \mathrm{CH}\right), 53.55$ $\left(\mathrm{COO}^{-} H_{2}\right), 59.64\left(\mathrm{CH}_{2} \underline{\mathrm{CH}}\right), 64.47\left(\underline{\mathrm{CPh}}_{3}\right), 116.82\left(\mathrm{CH}=\underline{\mathrm{CH}}_{2}\right), 126.63(\mathrm{Ar}), 127.91(\mathrm{Ar})$, 129.0 (Ar), 130.35 (Ar), $144.23\left(\underline{\mathrm{CH}}=\mathrm{CH}_{2}\right), 155.32\left(\mathrm{COOCH}_{2}\right), 171.75\left(\mathrm{C}(\mathrm{O}) \mathrm{NH}_{2}\right)$. IR $(\mathrm{KBr}): v=3317 \mathrm{~b}, 3030 \mathrm{~s}, 2926 \mathrm{~s}, 1682 \mathrm{~s}, 1232 \mathrm{~s}, 743 \mathrm{~s}, 701 \mathrm{~s} \mathrm{~cm}^{-1} \cdot[\alpha]^{20}{ }_{\mathrm{D}}=+23.8$ $\left(\mathrm{c}=1.0, \mathrm{CHCl}_{3}\right)$. HRMS (ESI): [M+ Na ${ }^{+}$, Calc: 469.1556, found: 469.1554 . 
To a stirred solution of the primary amide $35(2.74 \mathrm{~g}, 6.1 \mathrm{mmol})$ in dichloromethane $(20 \mathrm{~mL})$ at $0^{\circ} \mathrm{C}$ was added Lawesson's reagent $(1.49 \mathrm{~g}, 3.7 \mathrm{mmol})$, and the reaction mixture was stirred at $0^{\circ} \mathrm{C}$ for $1 \mathrm{~h}$ and at room temperature for $12 \mathrm{~h}$ (TLC control). The reaction mixture was concentrated and purified by column chromatography (silica gel, EtOAc/cyclohexane $=1: 2)$ to give $2.08 \mathrm{~g}(4.5 \mathrm{mmol}, 74 \%)$ of thioamide 22 as a colorless solid. M. p. $=124-125^{\circ} \mathrm{C}$. TLC: $R_{f}=0.28$ (EtOAc/cyclohexane $=1: 2$ ). ${ }^{1} \mathrm{H}-\mathrm{NMR}\left(400 \mathrm{MHz}, \mathrm{CD}_{3} \mathrm{CN}\right) \delta=2.57-2.69\left(2 \mathrm{H}, \mathrm{m}, \mathrm{CH}_{2}\right), 4.16,4.17(1 \mathrm{H}, \mathrm{d}, J=5.3$, $\left.\mathrm{CH}_{2} \mathrm{CH}\right), 4.49,4.499,4.50\left(2 \mathrm{H}, \mathrm{t}, J=1.8, \mathrm{COOCH}_{2}\right), 5.18,5.20(1 \mathrm{H}, \mathrm{d}, J=17.2$, $\left.\mathrm{CH}=\underline{\mathrm{CH}}_{2}\right), 5.28,5.32\left(1 \mathrm{H}, \mathrm{d}, J=17.2, \mathrm{CH}=\underline{\mathrm{CH}}_{2}\right), 5.81(1 \mathrm{H}, \mathrm{br}, \mathrm{NHCOO}), 5.87-5.96(1 \mathrm{H}$, $\left.\mathrm{m}, \underline{\mathrm{CH}}=\mathrm{CH}_{2}\right), 7.23-7.41(15 \mathrm{H}, \mathrm{m}$, trityl $), 7.76,8.05\left(2 \mathrm{H}, \mathrm{d}, J=116.5, \mathrm{NH}_{2}\right)$. ${ }^{13} \mathrm{C}-\mathrm{NMR}\left(100.6 \mathrm{MHz}, \quad \mathrm{CD}_{3} \mathrm{CN}\right) \delta=37.26\left(\underline{\mathrm{CH}}_{2} \mathrm{CH}\right), 60.49\left(\mathrm{COOCH}_{2}\right), 66.24$ $\left(\mathrm{CPh}_{3}\right), 67.72\left(\mathrm{CH}_{2} \underline{\mathrm{CH}}\right), 117.63\left(\mathrm{CH}=\underline{\mathrm{CH}}_{2}\right), 118.20(\mathrm{Ar}), 127.86(\mathrm{Ar}), 129.0(\mathrm{Ar}), 130.32$ (Ar), $134.05\left(\underline{\mathrm{CH}}=\mathrm{CH}_{2}\right), 145.48\left(\underline{\mathrm{COOCH}}_{2}\right), 207.56\left(\underline{\mathrm{C}}(\mathrm{S}) \mathrm{NH}_{2}\right) . \mathrm{IR}(\mathrm{KBr}): v=3299 \mathrm{~b}$, 3196 b, 2921 w, $1697 \mathrm{~s}, 1651 \mathrm{~s}, 1505 \mathrm{~s}, 891 \mathrm{~b}, 799 \mathrm{~s} \mathrm{~cm}^{-1} \cdot[\alpha]^{20}{ }_{\mathrm{D}}=+17.3(\mathrm{c}=1.0$, $\mathrm{CHCl}_{3}$ ). HRMS (ESI), [M+ $\left.\mathrm{Na}^{+}\right]$, Calc: 485.1328, found: 485.1325. Anal. calcd for $\mathrm{C}_{26} \mathrm{H}_{26} \mathrm{~N}_{2} \mathrm{O}_{2} \mathrm{~S}_{2}$ : C, 67.50; H, 5.66; N, 6.06; found: C, 66.8; H, 5.9; N, 5.8.

\section{Enantiomeric excess (e.e.) determination of building block 23 .}

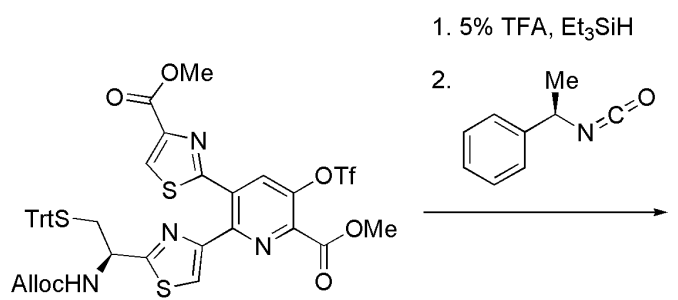

23

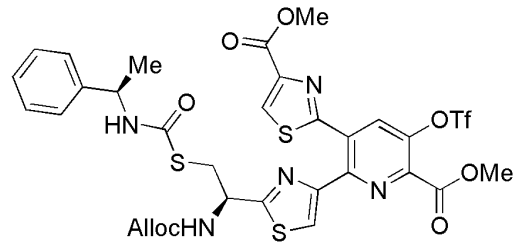

23a

Trifluoroacetic acid $(10 \mu \mathrm{L})$ was added dropwise to a stirred solution of trityl thioether $\mathbf{2 3}$ $(9.7 \mathrm{mg}, 0.01 \mathrm{mmol})$ in dry dichloromethane $(2 \mathrm{~mL})$ at room temperature, then triethylsilane $(6 \mu \mathrm{L})$ was added to the reaction mixture. The reaction mixture was stirred for $30 \mathrm{~min}$ (TLC control) and concentrated to dryness.

(R)-phenylethyl isocyanate $(1.8 \mu \mathrm{L}, 0.012 \mathrm{mmol})$ and pyridine $(1 \mu \mathrm{L})$ were added to the above residue in dichloromethane $(1 \mathrm{~mL})$ at room temperature. The reaction mixture was 
stirred for $10 \mathrm{~h}$ (TLC control), quenched by water $(1 \mathrm{~mL})$ and diluted by brine $(10 \mathrm{~mL})$, extracted with dichloromethane $(3 \times 10 \mathrm{~mL})$, dried with $\mathrm{Na}_{2} \mathrm{SO}_{4}$ and concentrated to dryness. Purification by column chromatography (silica gel, EtOAc/cyclohexane $=1: 3$ ) to give $4.2 \mathrm{mg}(0.005 \mathrm{mmol}, 48 \%)$ of urea $23 \mathrm{a}$ as a colorless solid. TLC: $R_{f}=0.21$ $($ EtOAc/cyclohexane $=1: 2)$; d.e. $>85 \%$.

\section{${ }^{1} \mathrm{H}-\mathrm{NMR}$ of 23a.}

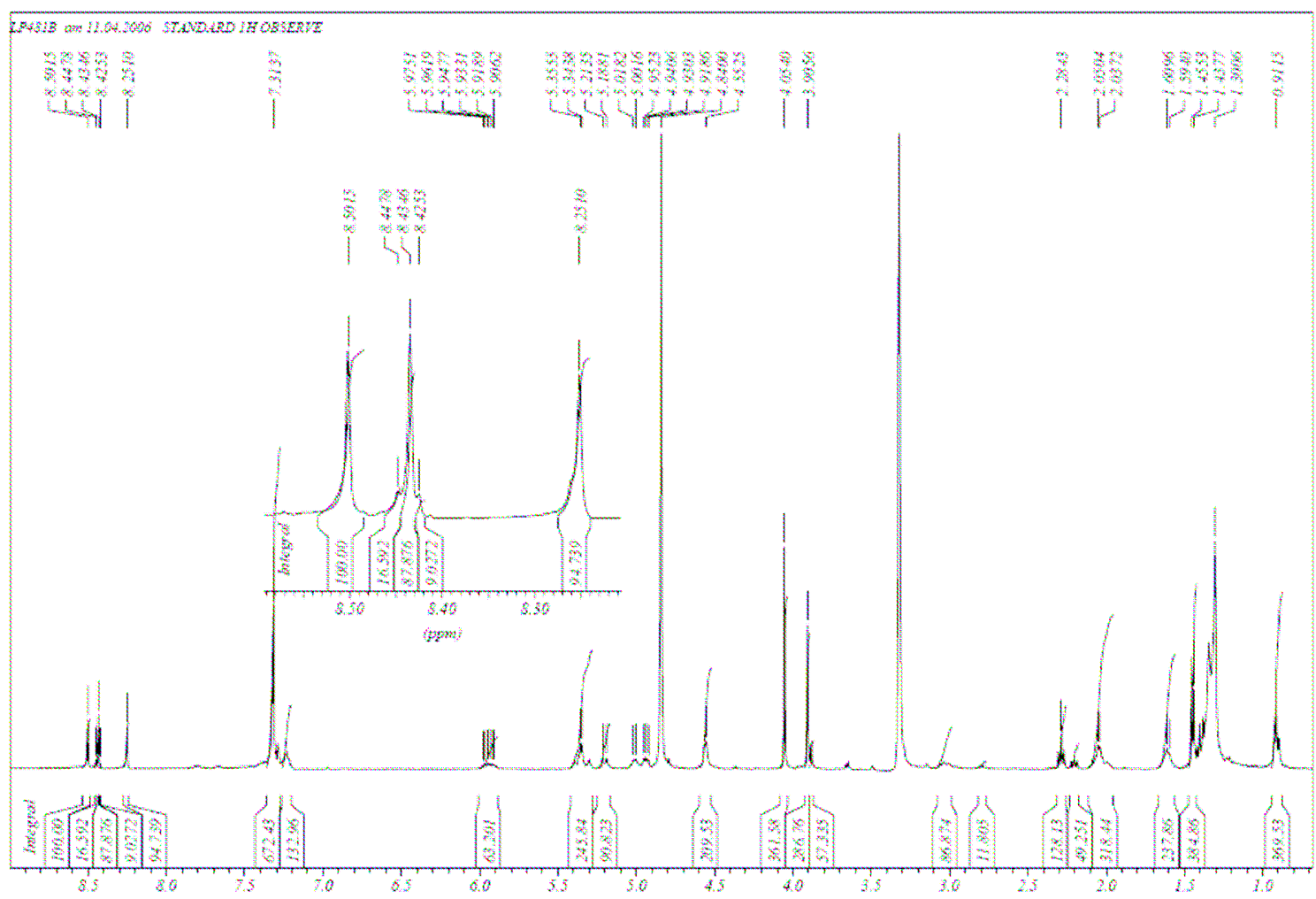

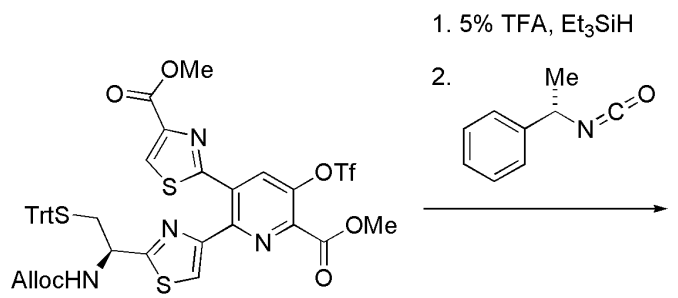

23

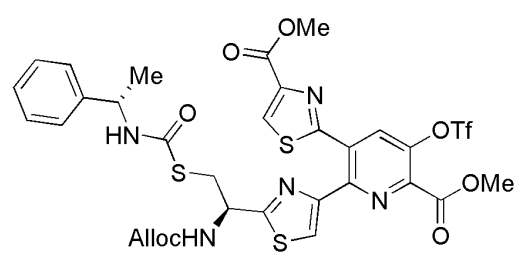

23b

23b was obtained likewise from ( $S$ )-phenylethyl isocyanate (54\% yield): colorless solid; d.e. $>60 \%$. 
${ }^{1} \mathrm{H}-\mathrm{NMR}$ of $\mathbf{2 3 b}$.

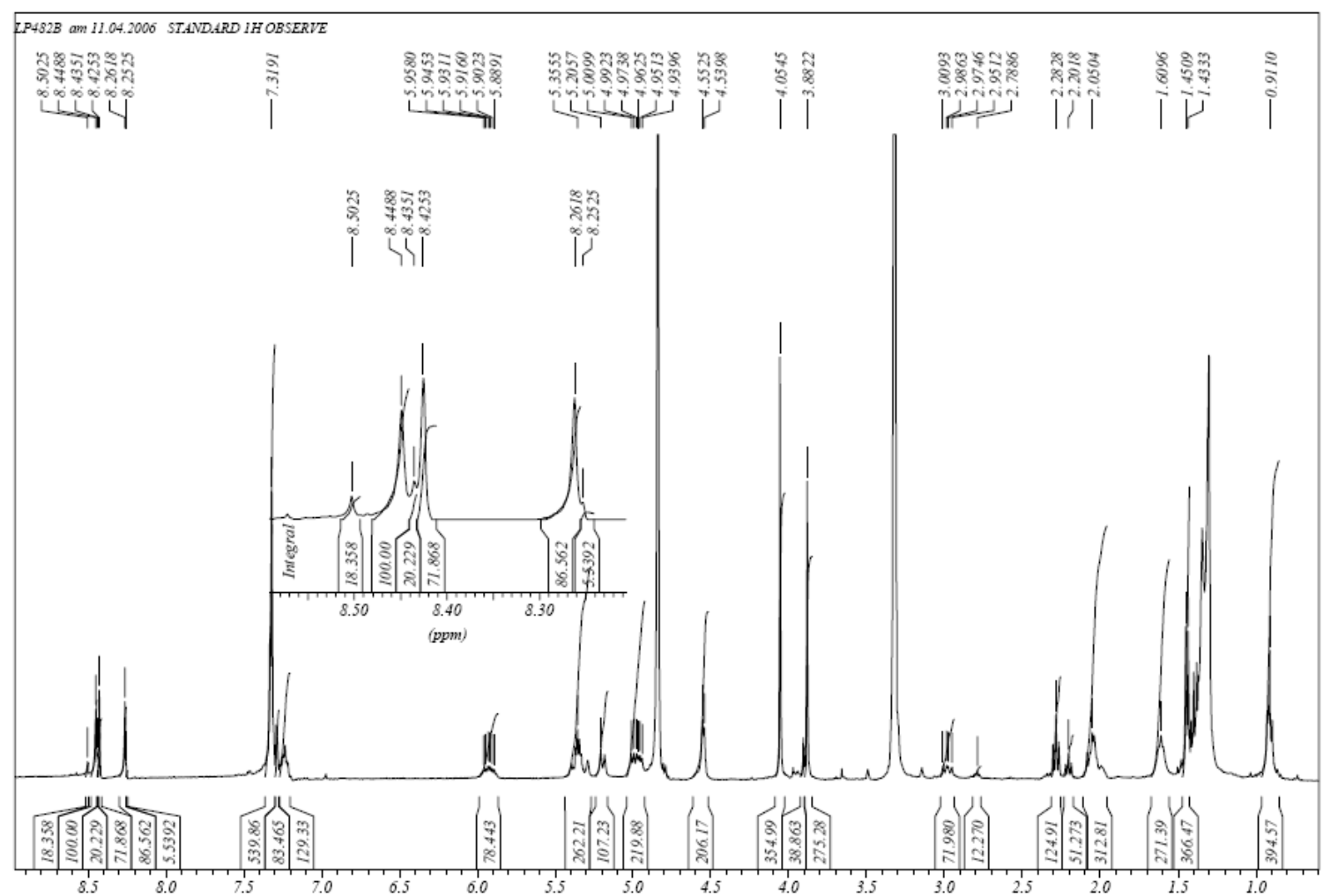

Enantiomeric excess (e.e.) determination of building block 25a.

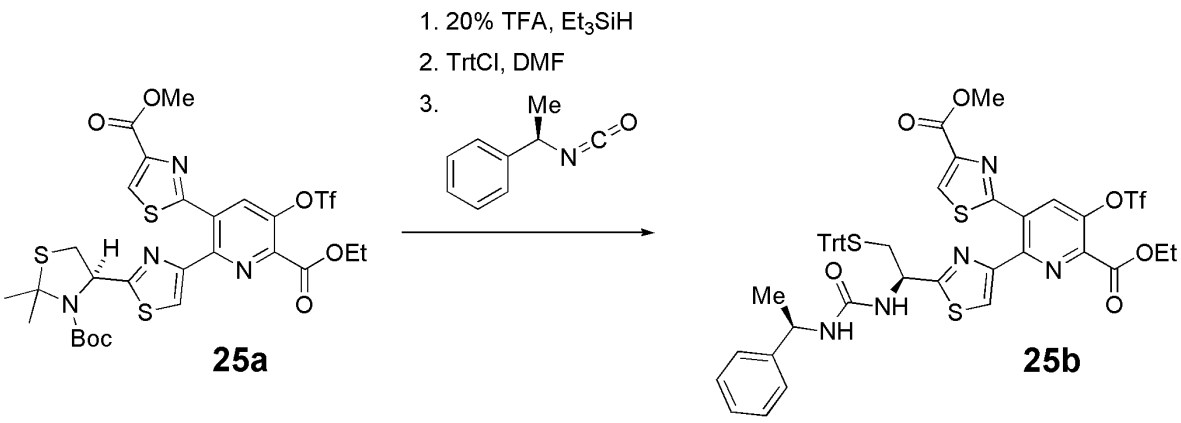

Trifluoroacetic acid $(0.6 \mathrm{~mL})$ was added dropwise to a stirred solution of thioaminal $25 \mathbf{a}$ $(24 \mathrm{mg}, 0.03 \mathrm{mmol})$ in dry dichloromethane $(3 \mathrm{~mL})$ at room temperature, then triethylsilane $(0.6 \mathrm{~mL})$ was added to the reaction mixture. The reaction mixture was stirred for $1 \mathrm{~h}$ (TLC control) and concentrated to dryness. (MALDI_TOF), [M $\left.+\mathrm{H}^{+}\right]$, calc: 599.0 , found: 599.7 . 
Trityl chloride (13.6 mg, $0.05 \mathrm{mmol}$ ) was added to the above residue in DMF (2 mL) at room temperature and the reaction mixture was stirred for 2 days. The reaction mixture was diluted by dichloromethane $(60 \mathrm{~mL})$, washed by $10 \%$ sodium acetate solution $(2 \mathrm{x}$ $20 \mathrm{~mL})$ and brine $(2 \times 20 \mathrm{~mL})$, the organic layer was dried with $\mathrm{Na}_{2} \mathrm{SO}_{4}$ and concentrated to dryness. Purification by column chromatography (silica gel, EtOAc/light petroleum = $1: 1)$ to give $18 \mathrm{mg}(0.02 \mathrm{mmol}, 67 \%)$ of the $S$-trityl aminothiol. TLC: $R_{f}=0.28$ (EtOAc/light petroleum $=1: 1$ ), (MALDI_TOF), $\left[\mathrm{M}+\mathrm{H}^{+}\right]$, calc: 841.1, found: 842.0. $(R)$-phenylethyl isocyanate $(5 \mu \mathrm{L}, 0.033 \mathrm{mmol})$ and pyridine $(2.7 \mu \mathrm{L})$ were added to the $S$-trityl aminothiol $(9 \mathrm{mg}, 0.01 \mathrm{mmol})$ in dichoromethane $(1 \mathrm{~mL})$ at room temperature. The reaction mixture was stirred for $3 \mathrm{~h}$, quenched with water $(1 \mathrm{~mL})$, diluted by brine $(10 \mathrm{~mL})$ and extracted with dichloromethane $(3 \times 10 \mathrm{~mL})$. The organic extracts were dried with $\mathrm{Na}_{2} \mathrm{SO}_{4}$ and concentrated. Purification by column chromatography (silica gel, EtOAc/light petroleum = $1: 4)$ to give $4.0 \mathrm{mg}(0.004 \mathrm{mmol}, 40 \%)$ of the urea $25 \mathbf{b}$ as a colorless solid. TLC: $R_{f}=0.37$ (EtOAc/light petroleum $=1: 1$ ). (MALDI_TOF), [M + $\mathrm{Na}^{+}$], calc: 1010.2 , found: 1010.9 ; d.e. $>99 \%$.

${ }^{1} \mathrm{H}-\mathrm{NMR}$ of $\mathbf{2 5 b}$.

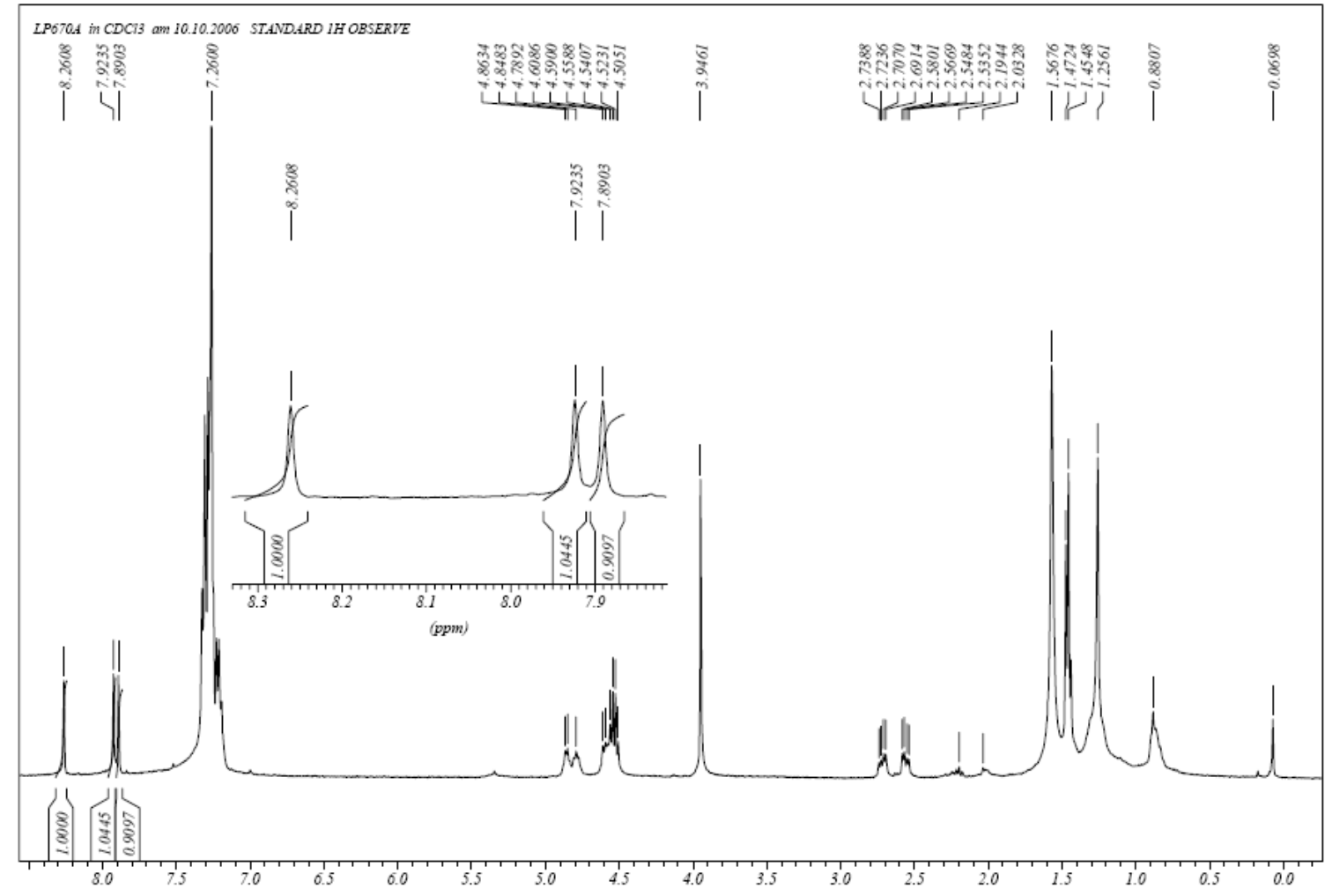




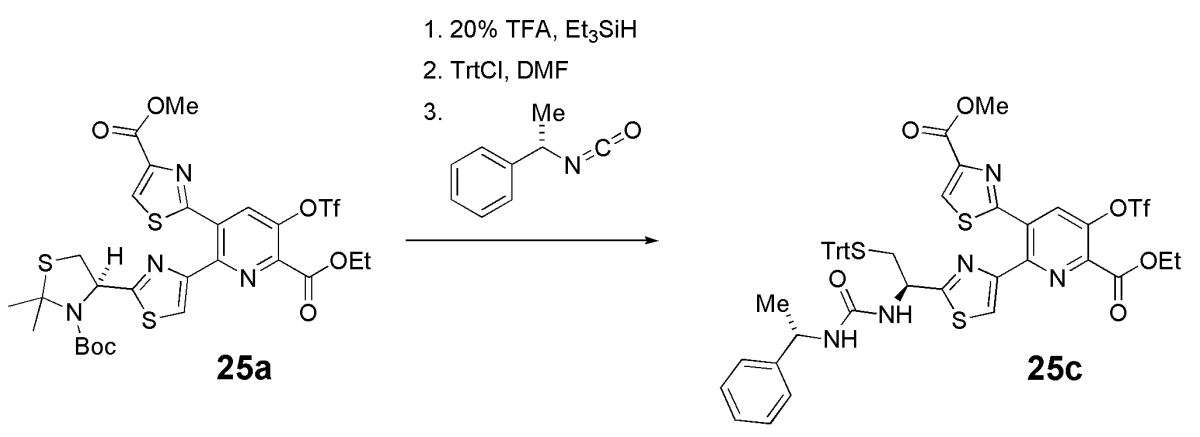

25c was obtained likewise from $(S)$-phenylethyl isocyanate (50\% yield) as a colorless solid. TLC: $R_{f}=0.37($ EtOAc/light petroleum $=1: 1)$. (MALDI_TOF), [M+ $\left.\mathrm{Na}^{+}\right]$, calc: 1010.2, found: 1010.9. d.e. $>96 \%$.

\section{${ }^{1} \mathrm{H}-\mathrm{NMR}$ of $25 \mathrm{c}$.}

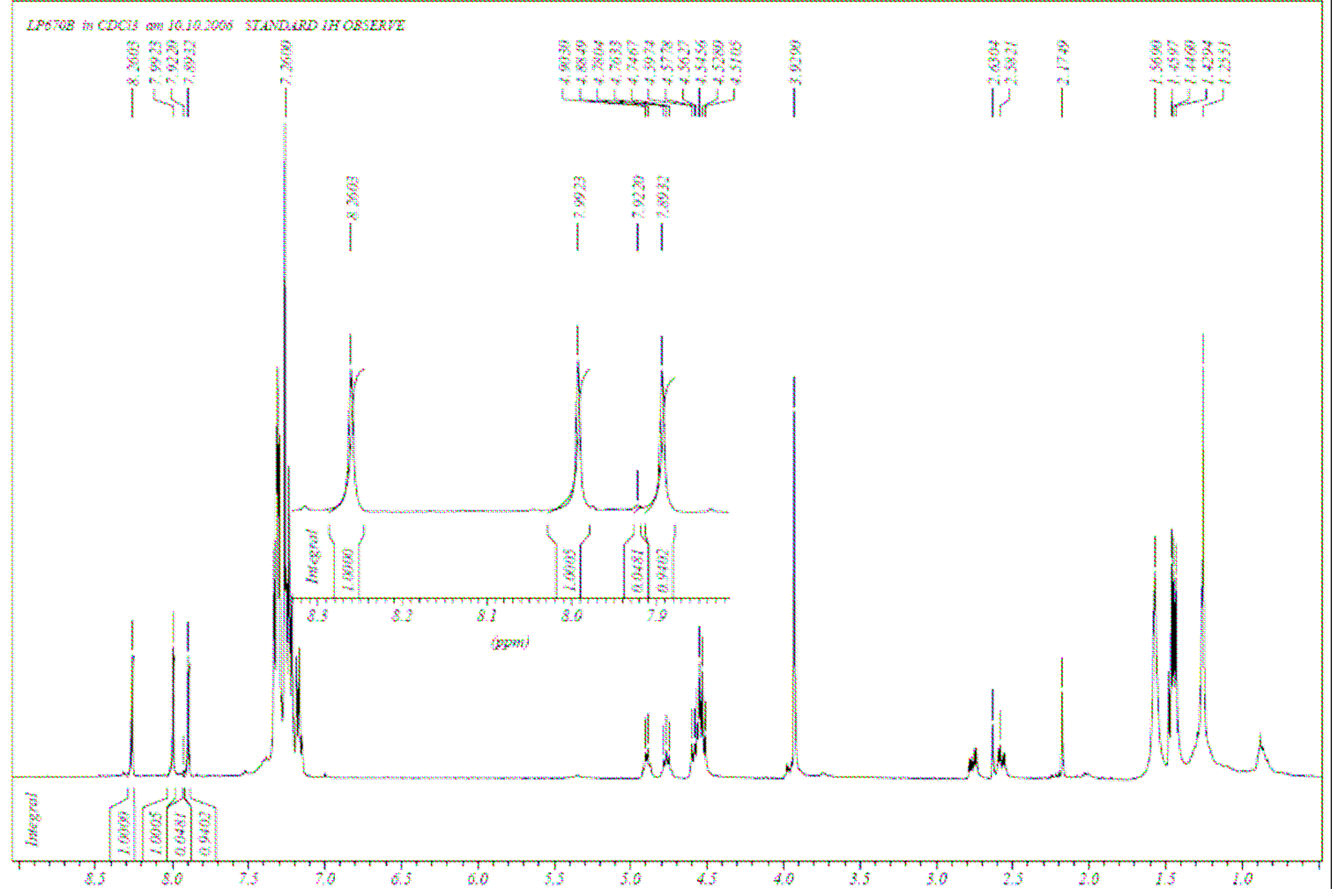


${ }^{1} \mathrm{H}-\mathrm{NMR}$ of $\mathbf{2 5 b}$ and $\mathbf{2 5 c}$ after mixing:

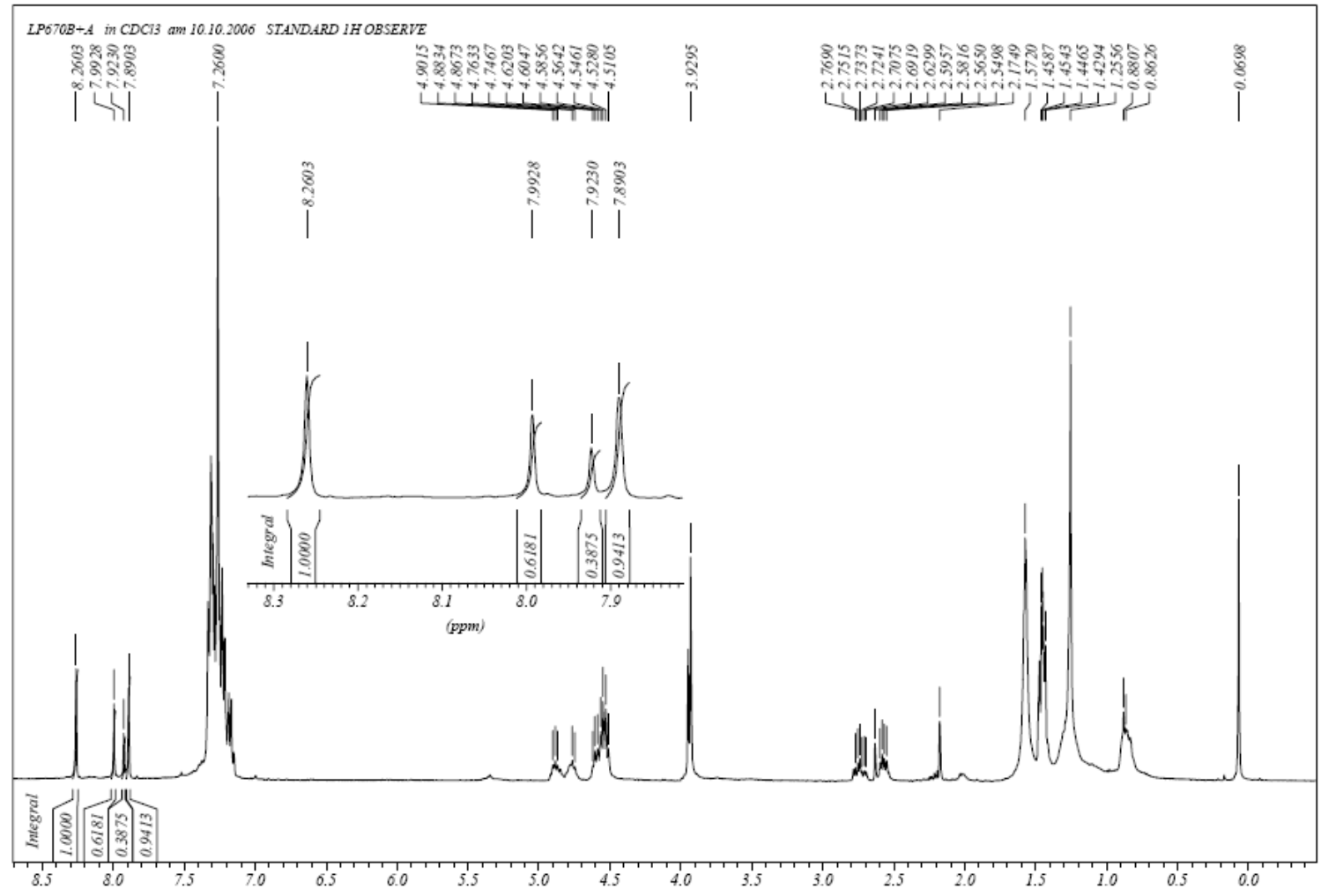

\section{References:}

1 Armarego, W. L. F.; Chai, C. L. L. Purification of Laboratory Chemicals, 5th Ed, Elsevier, 2002.

2 Igarashi, J. -E.; Kawakami, Y.; Kinoshita, T.; Furukawa, S. Chem. Pharm. Bull. 1990, 38, 1832.

3 Li, L.-S.; Wu, Y.-L. Tetrahedron Lett. 2002, 43, 2427.

4 Barluenga, J.; Rodríguez, M. A.; Campos, P. J. Tetrahedron Lett. 1990, 31, 2751.

5 Kelly, T. R.; Lang, F. J. Org. Chem. 1996, 61, 4623. 


\section{${ }^{1} \mathrm{H}$-spectra of compounds 6-25.}

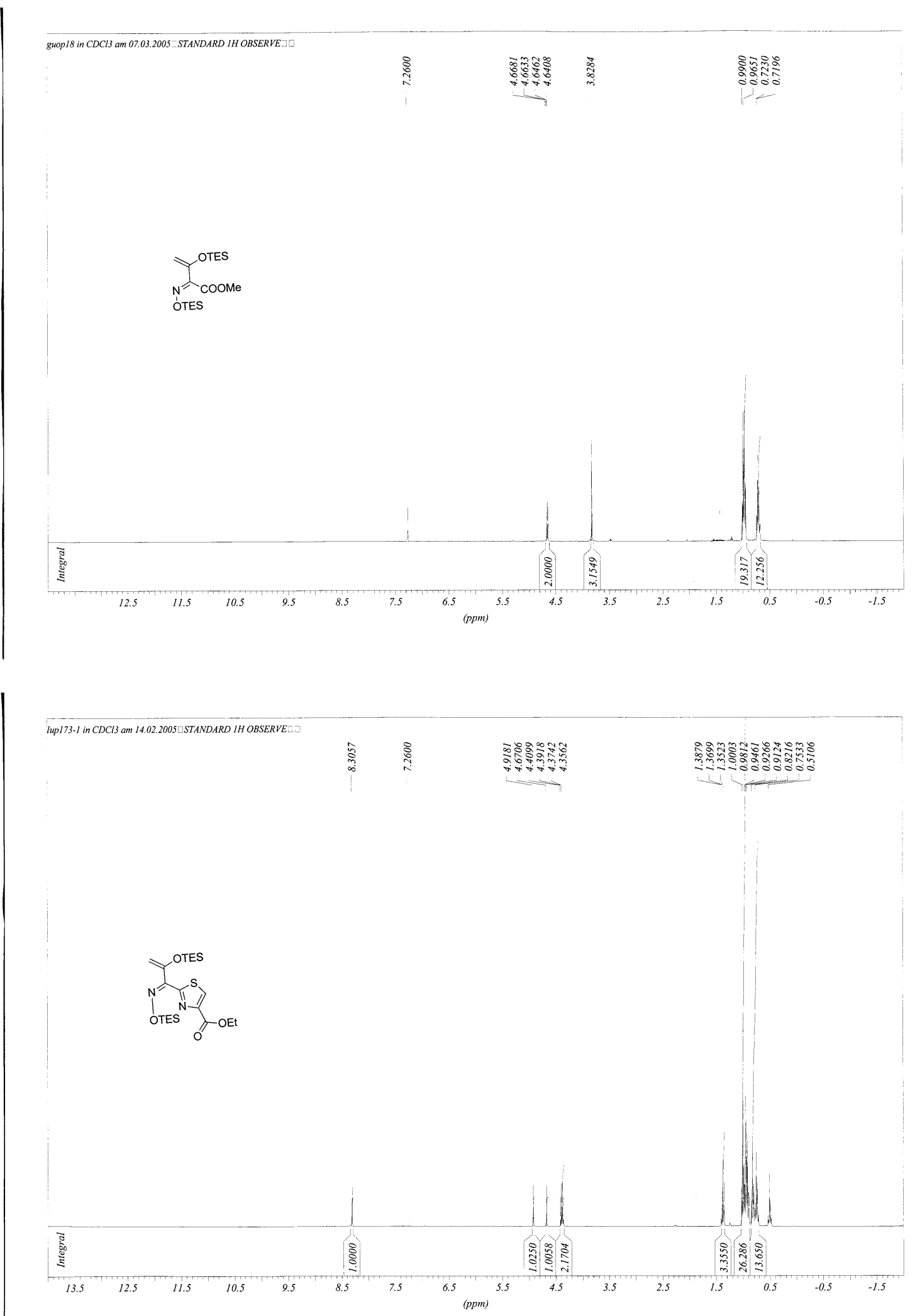




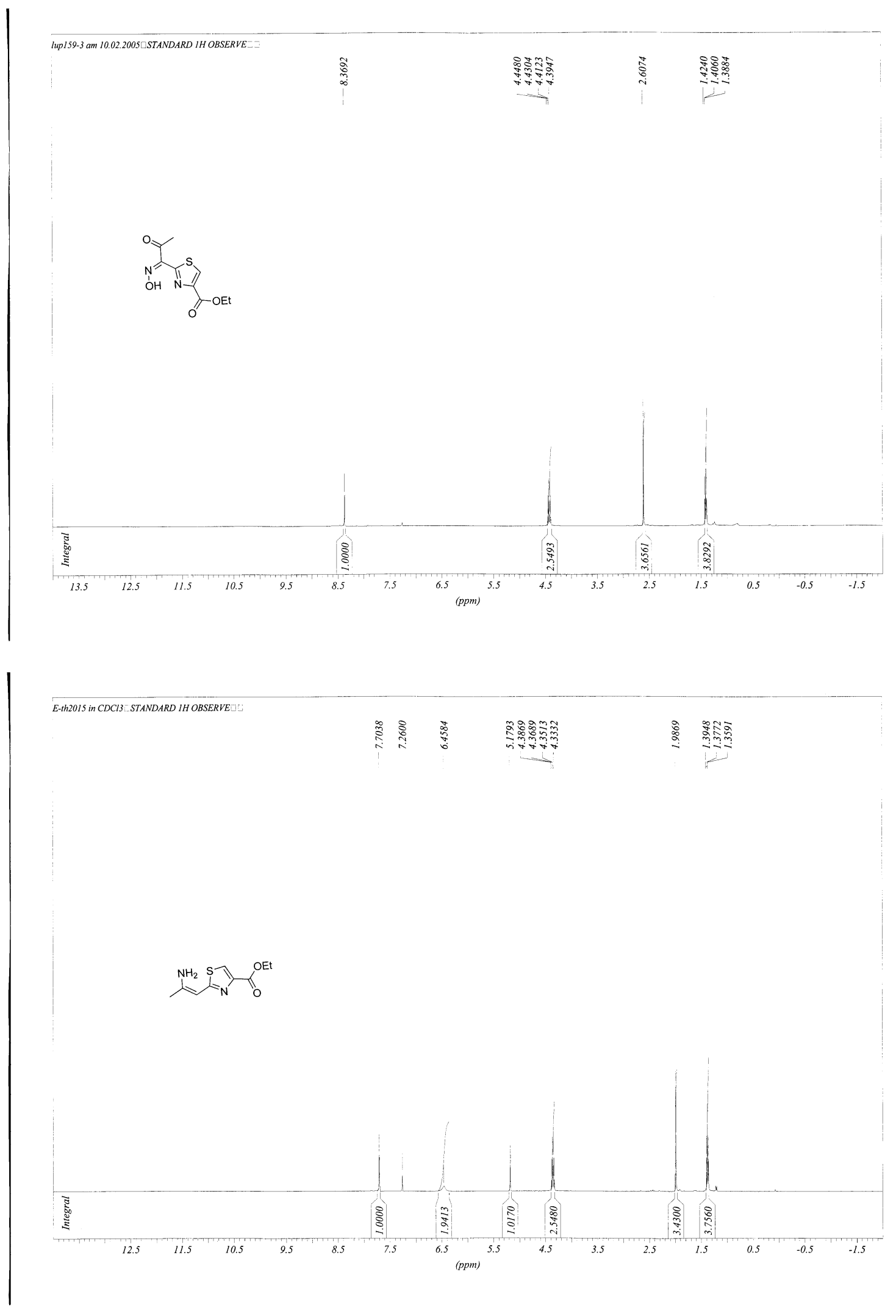



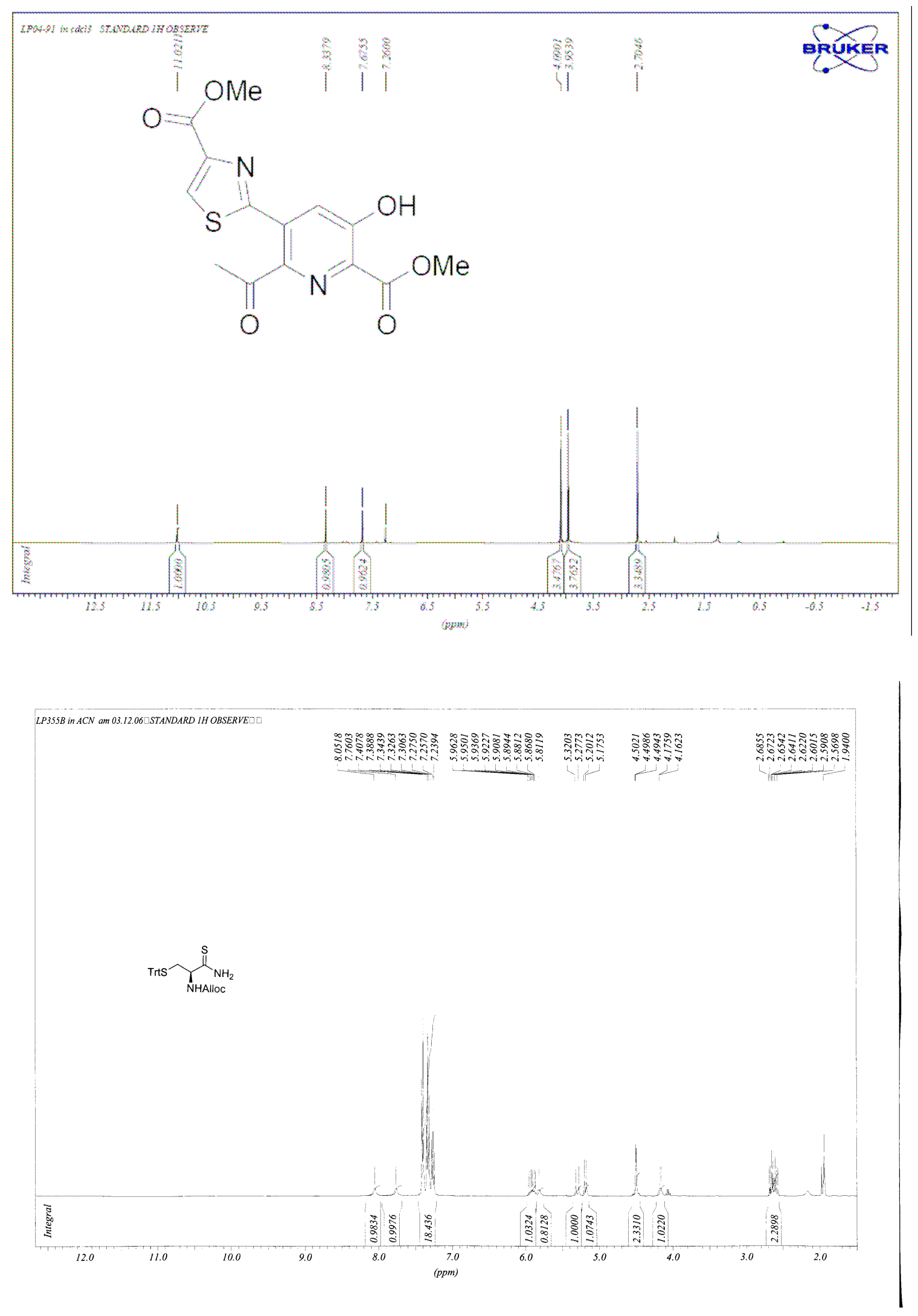


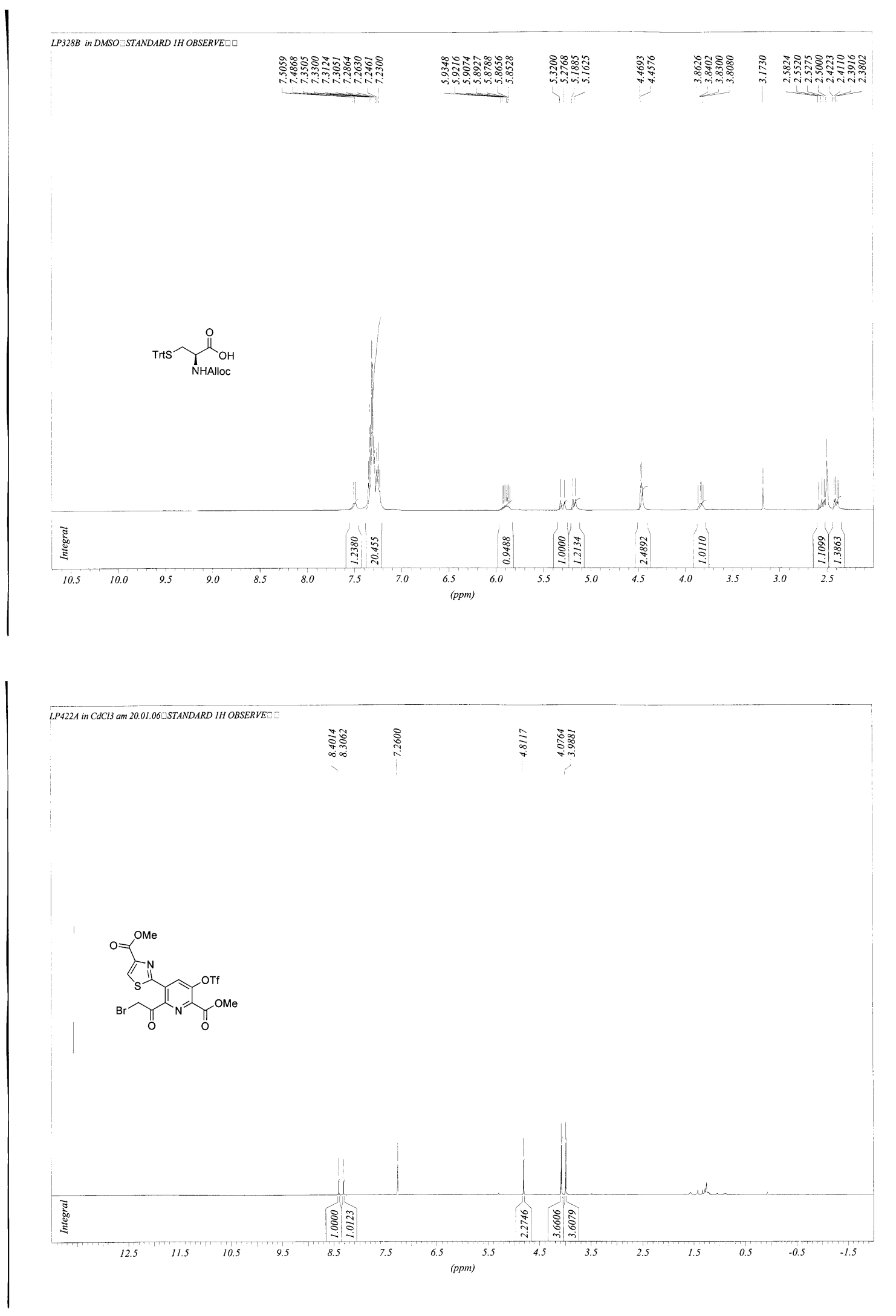




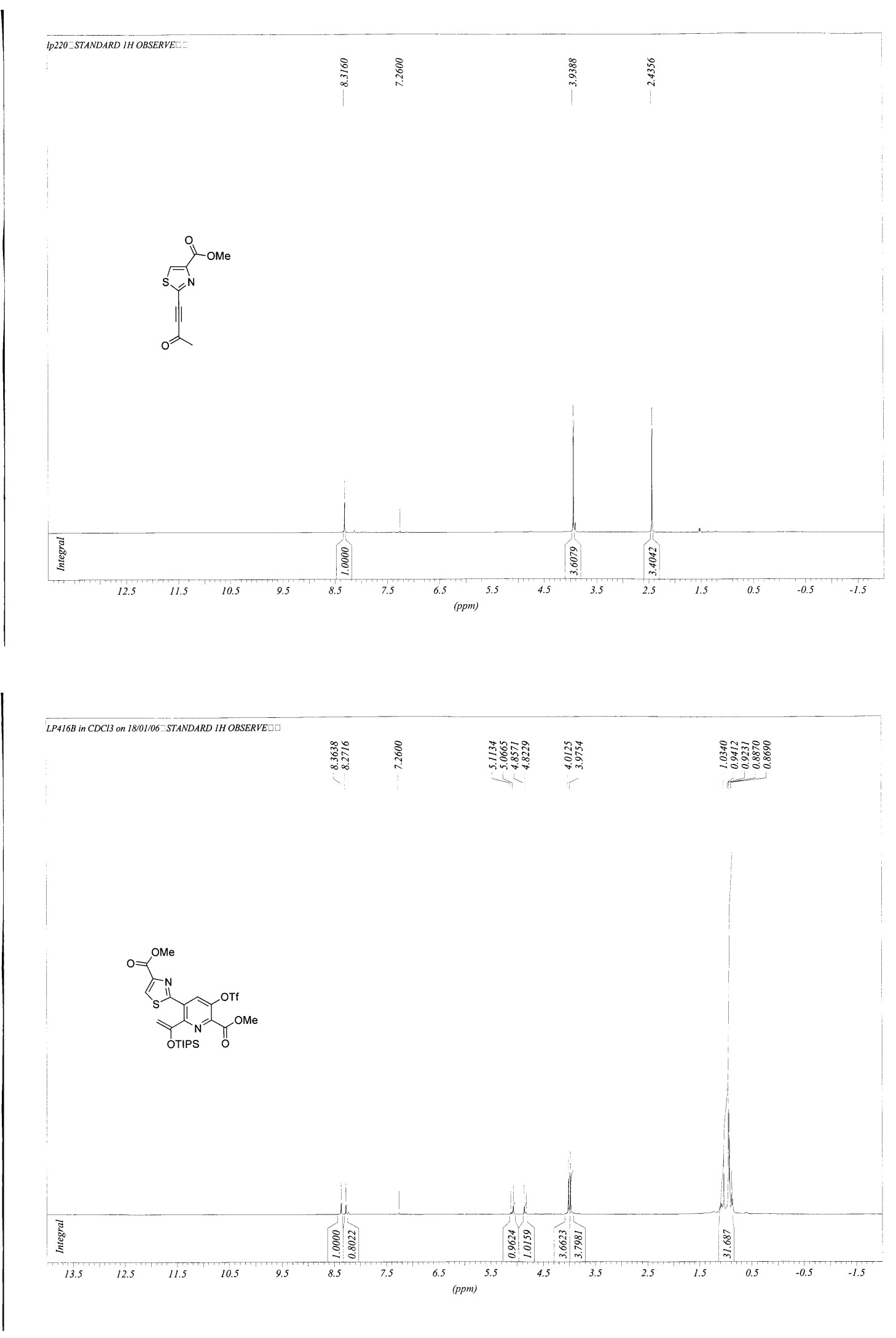




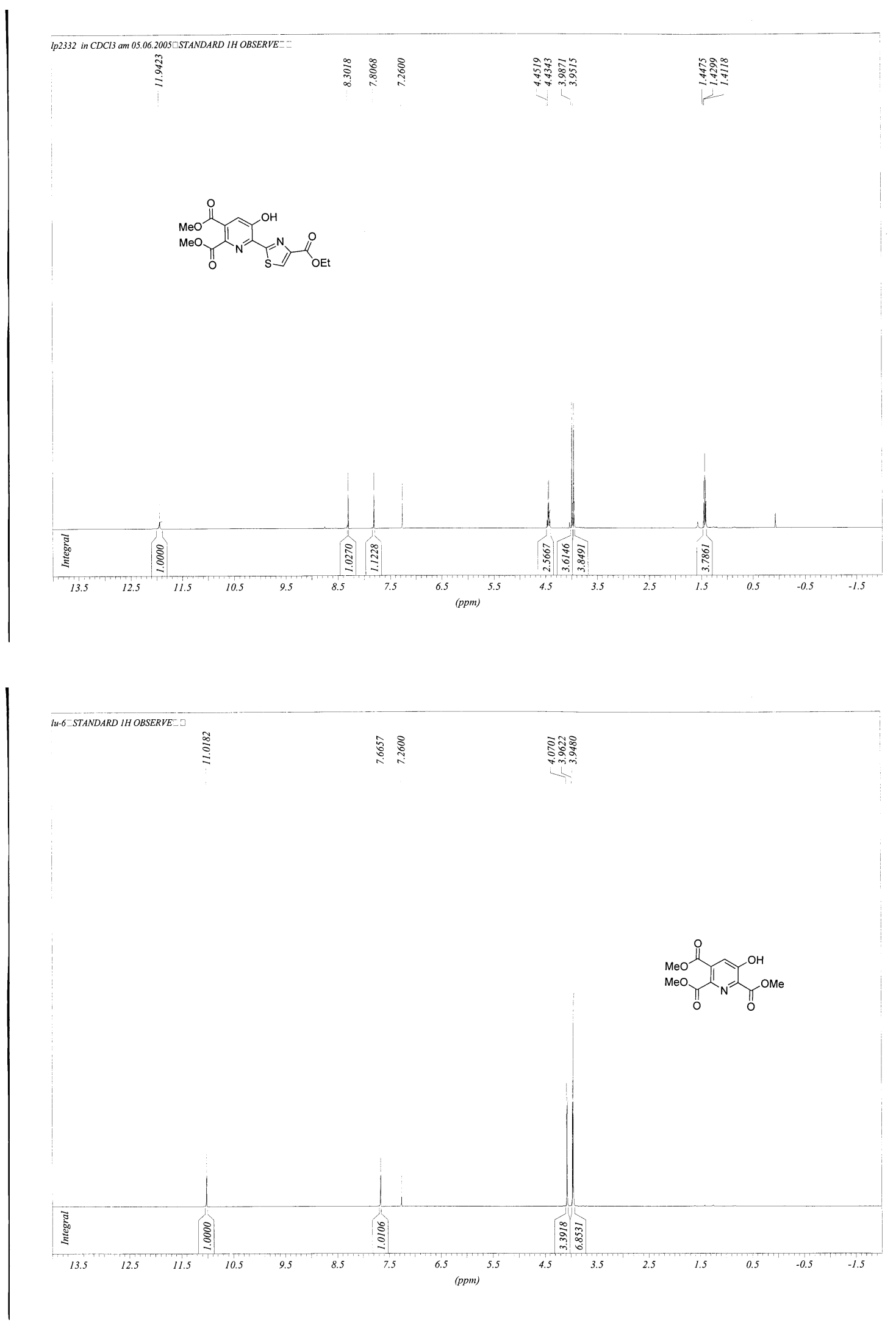



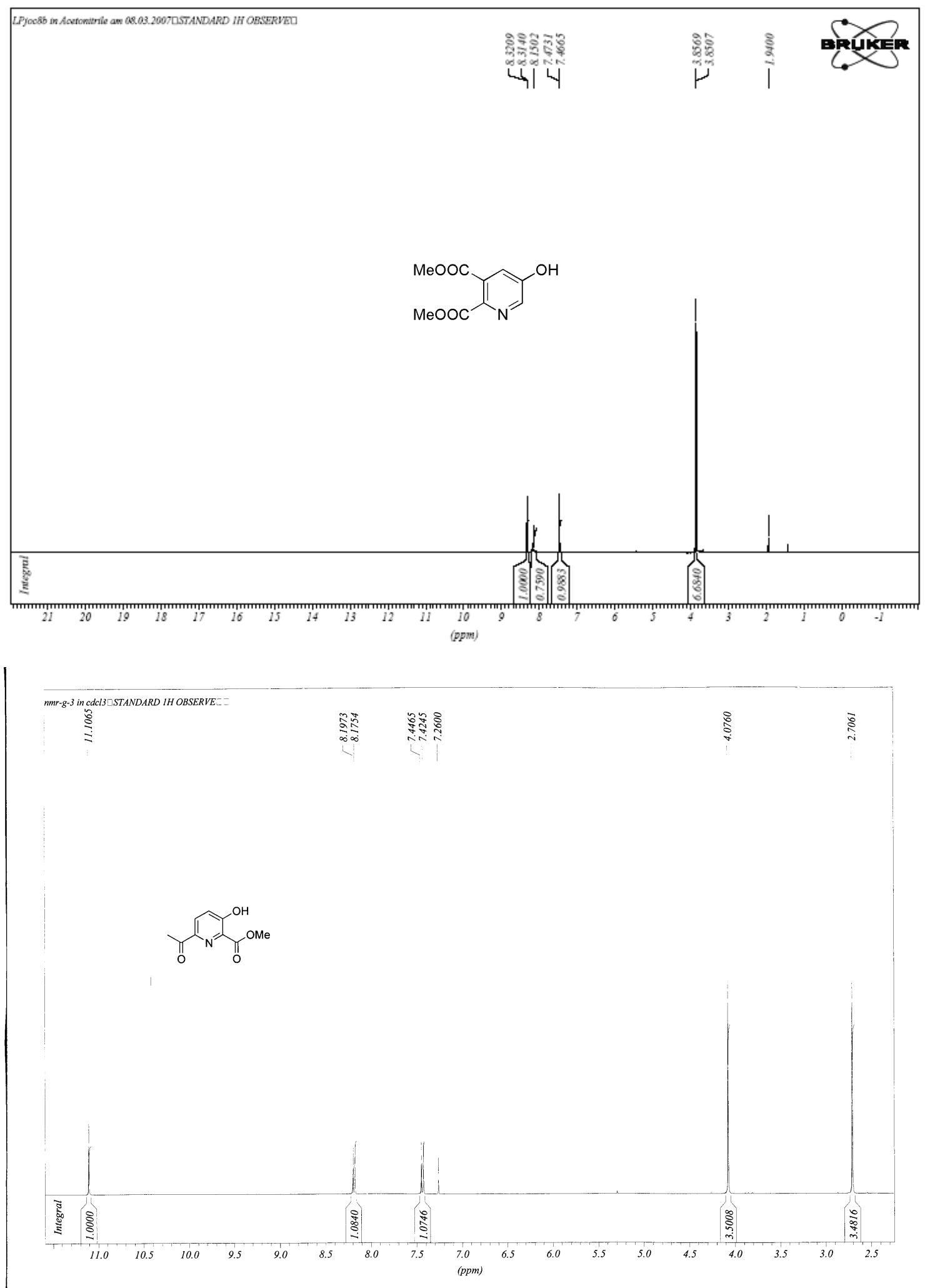

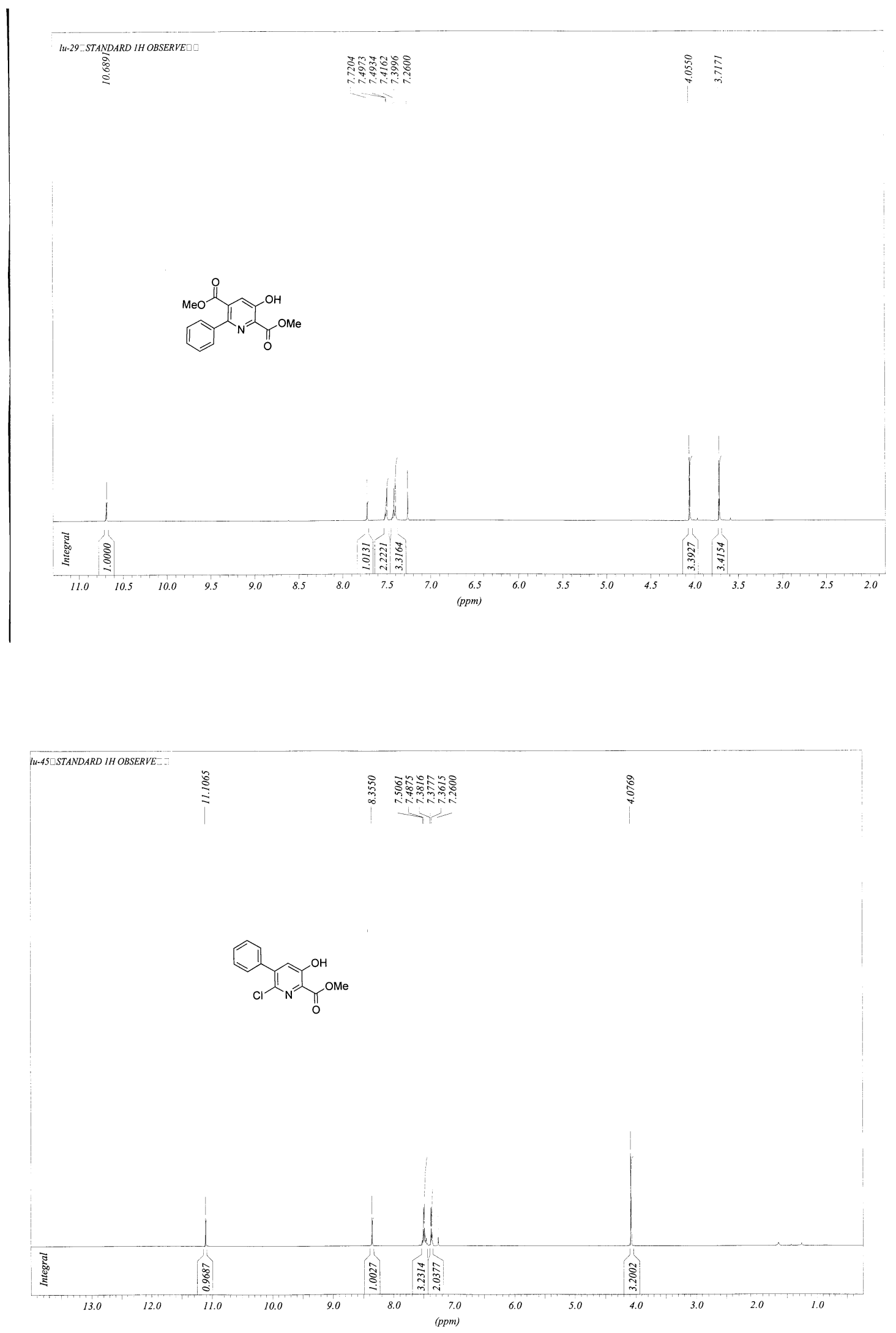

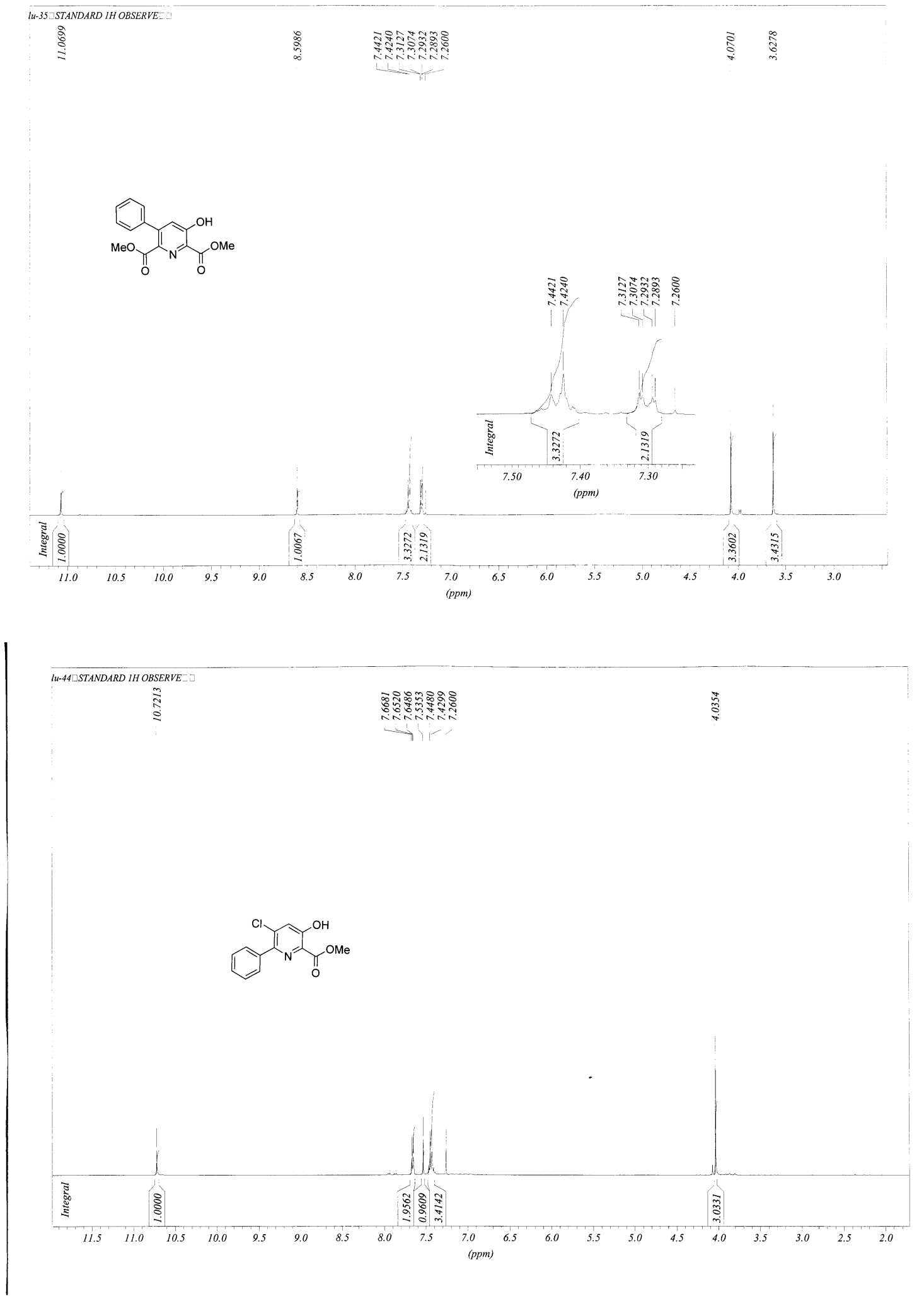


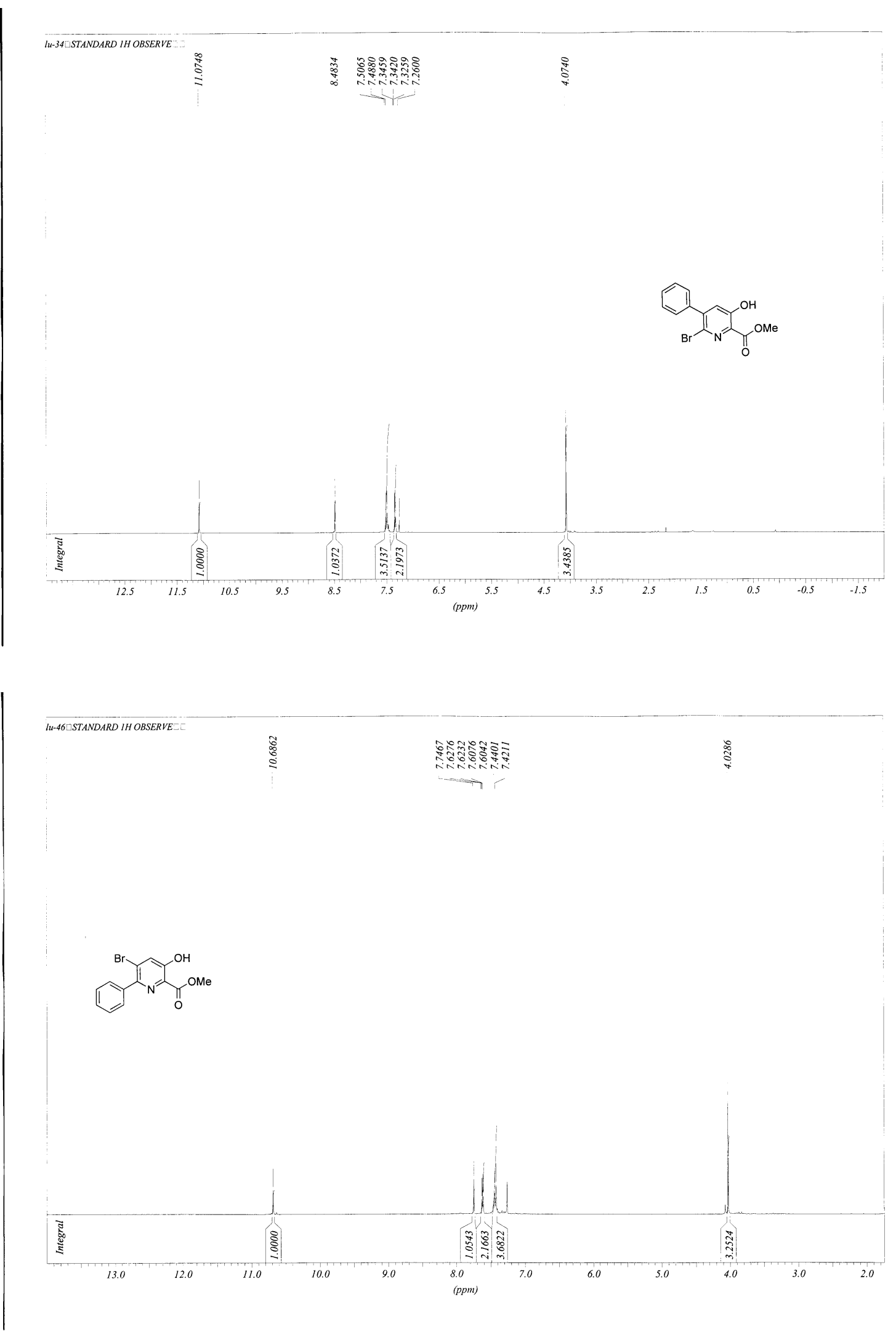



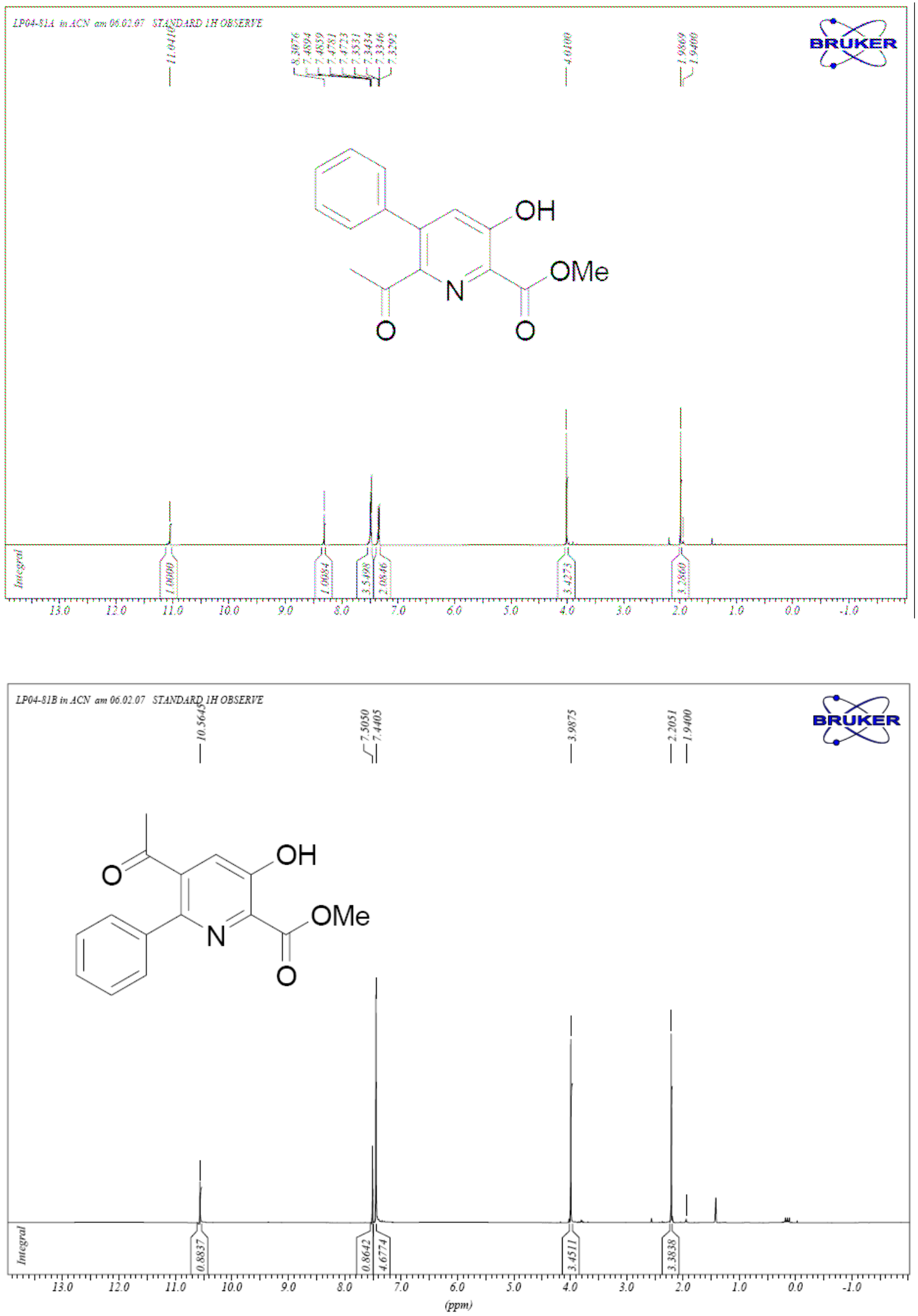

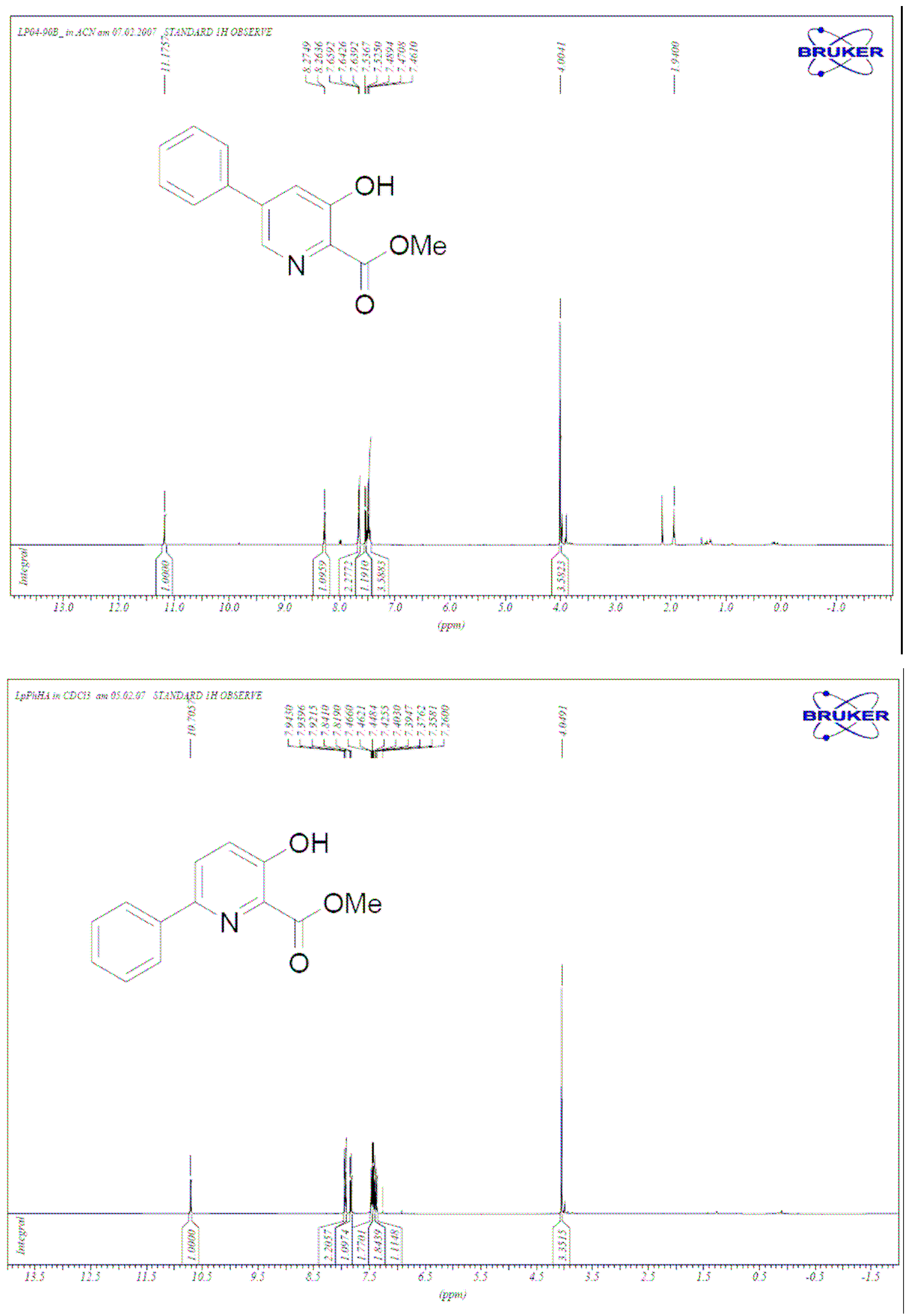

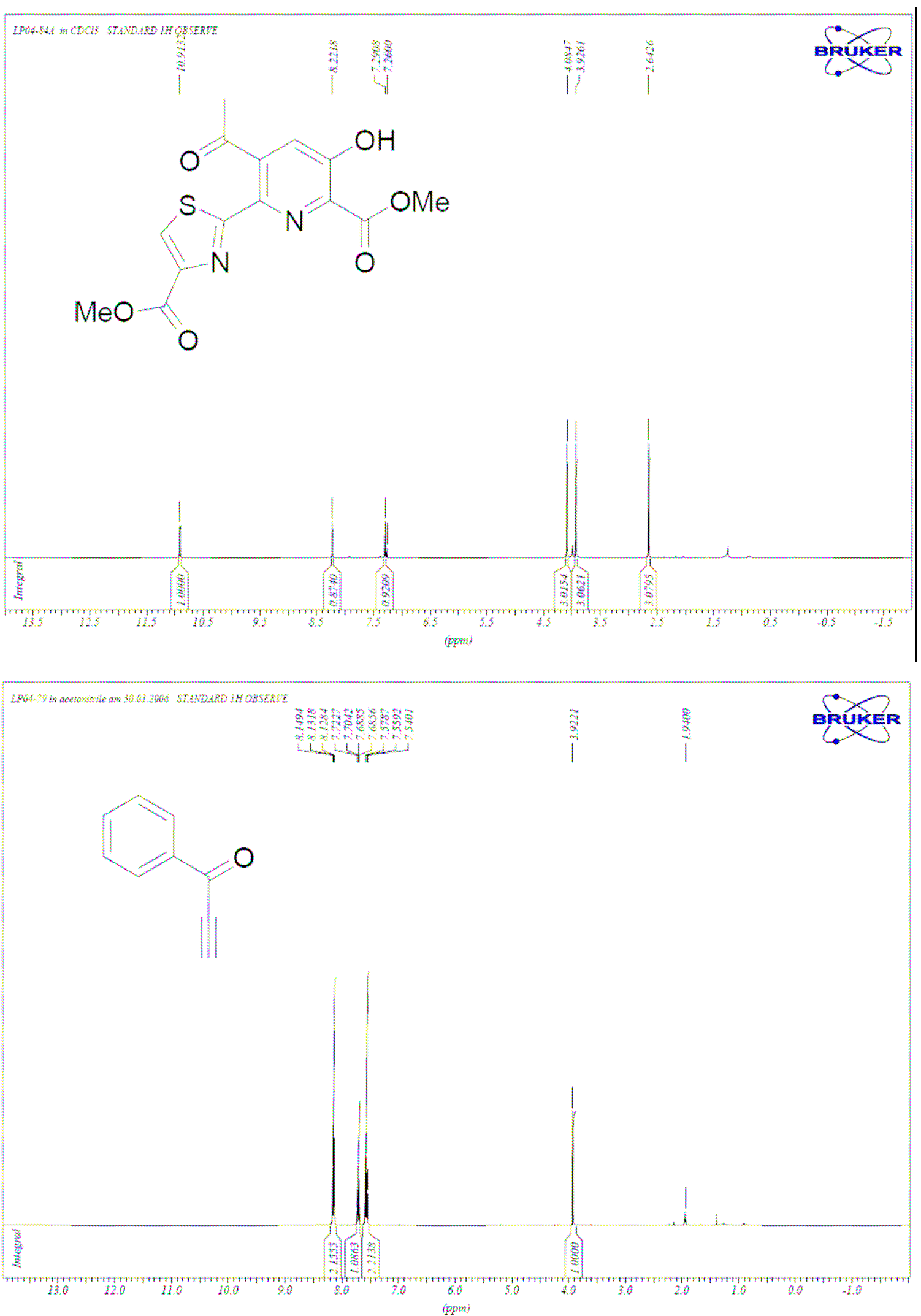

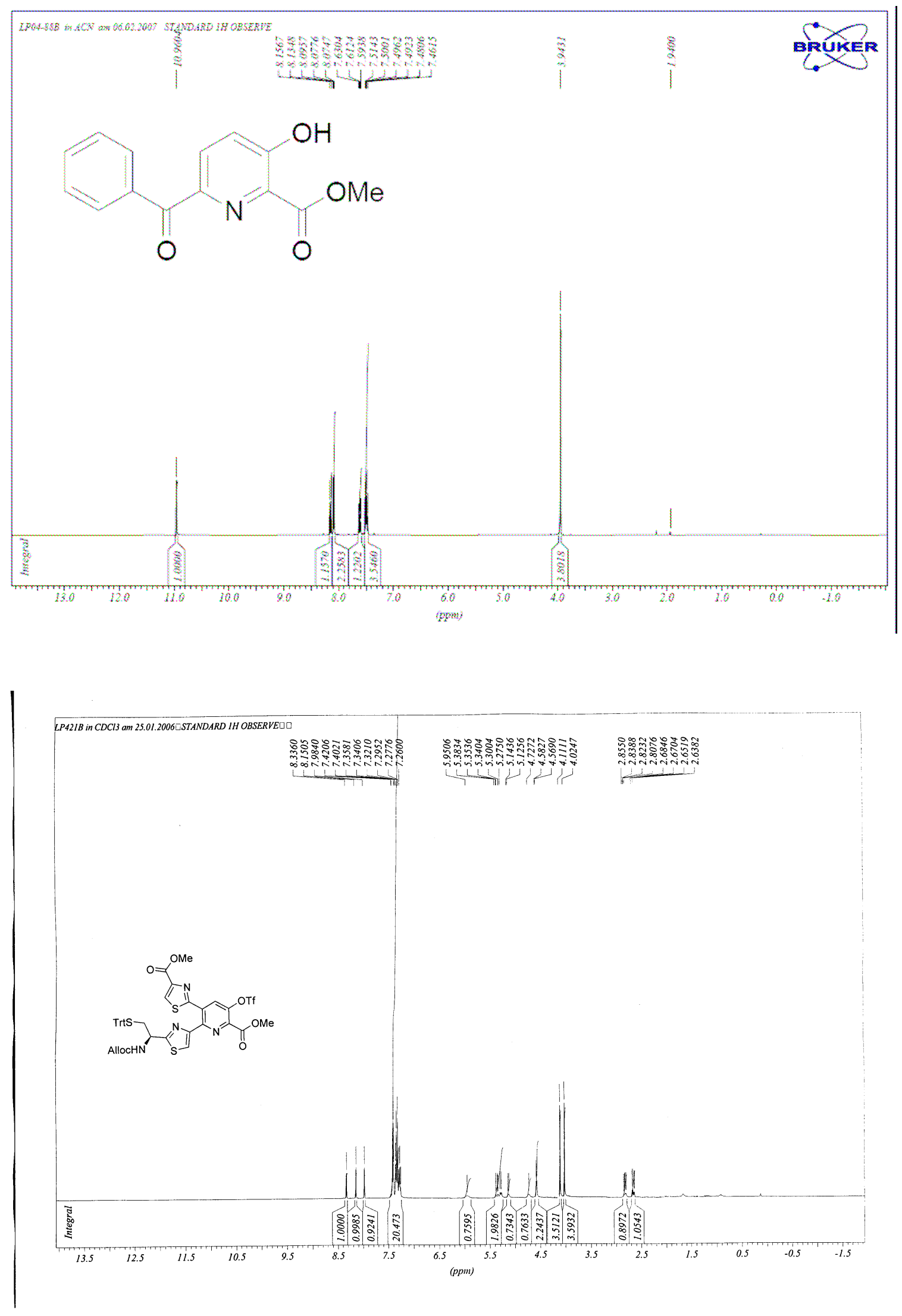


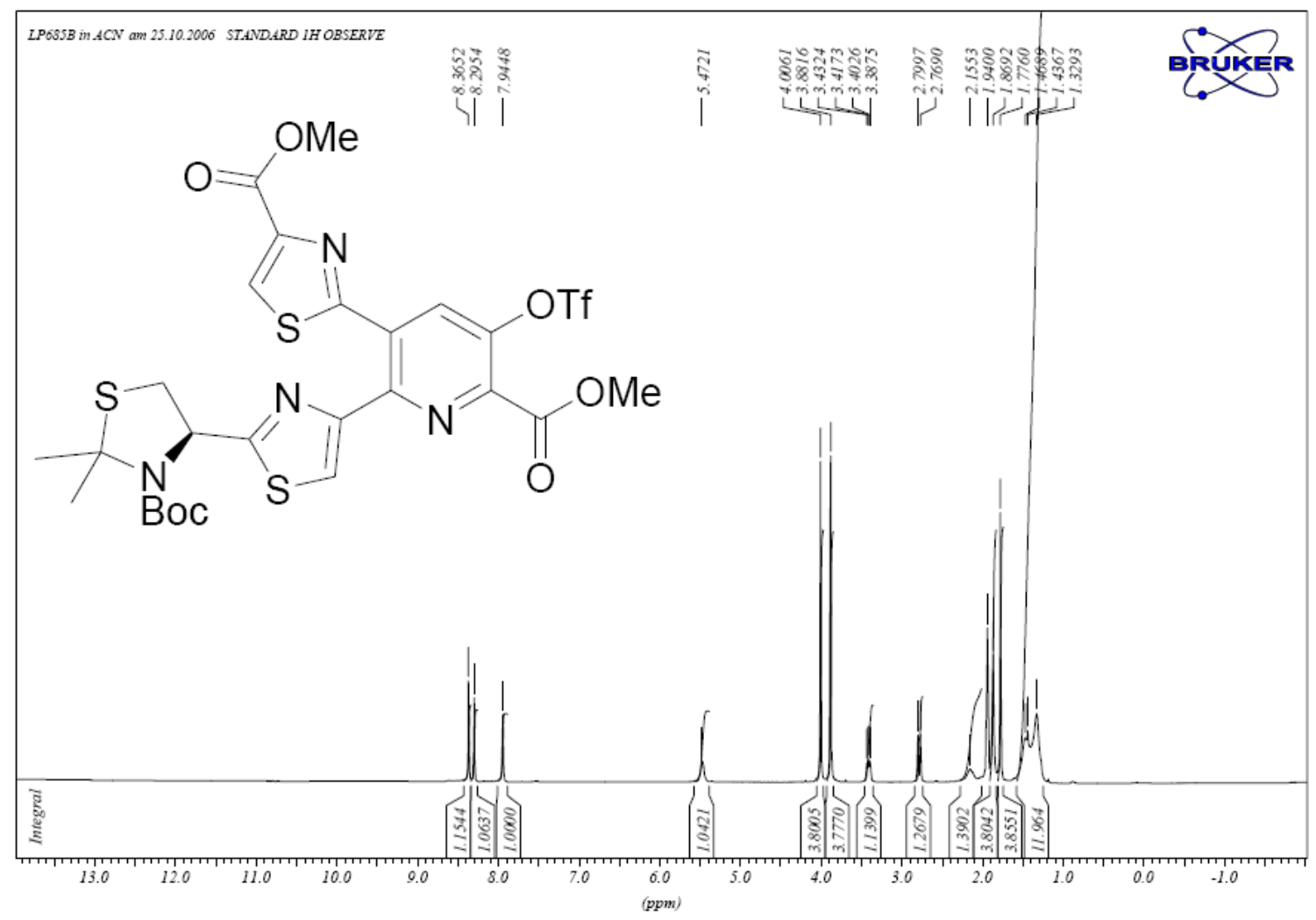

